\title{
Lösungsorientierte Fragen im Coaching- und Psychotherapie-Gespräch - Ein gesprächslinguistischer Vergleich ihres interaktionstypspezifischen, lokalen Veränderungspotentials
}

\author{
Susanne Kabatnik ${ }^{1}$ D . Eva-Maria Graf ${ }^{2}$ \\ Angenommen: 2. November 2021 / Online publiziert: 6. Dezember 2021 \\ (c) Der/die Autor(en) 2021
}

\section{Zusammenfassung}

Der Beitrag thematisiert Lösungsorientierte Fragen (LoF) in Coaching und Psychotherapie aus linguistischer Sicht. Basierend auf gesprächsanalytischen Vorarbeiten wird die Interaktionstypspezifik von Lösungsorientierten Fragen im Coaching und in der Psychotherapie komparativ (re-)analysiert und dokumentiert. Dazu werden die formalen, funktionalen, kontextuellen und Coaching-spezifischen Charakteristika Lösungsorientierter Fragen beschrieben und es wird aufgezeigt, wie LoF zur lokalen Veränderung im Coaching-Gespräch beitragen. Parallel dazu werden diese Ergebnisse mit Erkenntnissen zu Lösungsorientierten Fragen aus dem Beratungsformat Psychotherapie verglichen und die Interaktionstyp-spezifischen Gemeinsamkeiten und Unterschiede vor dem Hintergrund der jeweiligen Handlungsrationale interpretiert. Eine erste Ausdifferenzierung des Fragetyps Lösungsorientierte Fragen für das Beratungsformat Coaching wird vorgeschlagen.

Schlüsselwörter Lösungsorientierte Fragen · Coaching · Psychotherapie · Linguistische Gesprächsanalyse · Interaktionstypspezifik · Lokales Veränderungspotential

\section{Solution-oriented questions in coaching and therapeutic conversations-analyzing their interaction-type specific local change potential from a linguistic perspective}

\begin{abstract}
This paper topicalizes solution-oriented questions in coaching and psychotherapy from a linguistic perspective. Based on preliminary studies in linguistischer Gesprächsanalyse on solution-oriented questions in both formats, we will comparatively (re-)analyze and document the interaction-type specificity of solution-oriented questions in coaching and psychotherapy. To this end, we will describe the formal, functional, contextual, and coaching-specific characteristics of solution-oriented questions in coaching, and we will show how they contribute to the local change of the coaching conversation. These results will be compared to findings from psychotherapy; overall interaction-type commonalities and differences are interpreted against the backdrop of the formats' underlying action rationale. We end with suggesting a first differentiation of solution-oriented questions for coaching.
\end{abstract}

Susanne Kabatnik

susanne.kabatnik@uni-greifswald.de

Eva-Maria Graf

eva-maria.graf@aau.at

1 Arbeitsbereich Germanistische Sprachwissenschaft, Institut für deutsche Philologie, Universität Greifswald, Rubenowstraße 3 - Raum 2.11, 17487 Greifswald, Deutschland
2 Institut für Anglistik und Amerikanistik, Universität Klagenfurt, Universitätsstraße 65-67, 9020 Klagenfurt am Wörthersee, Österreich 
Keywords Solution-oriented questions · Coaching · Psychotherapy · Linguistische Gesprächsanalyse · Interaction-type specificity $\cdot$ Local change potential

\section{Einleitung}

Lösungsorientierte Fragen implizieren begrifflich immer schon Veränderung. Ein wie auch immer geartetes Problem, eine Fragestellung oder gar ein Konflikt soll gedanklich einer Lösung zugeführt und der gegenwärtige Status verändert werden. Grundlage dafür bilden Struktureigenschaften von Kommunikation. (Kabatnik et al. 2019, S. 147).

Der vorliegende Beitrag fokussiert und kontrastiert Lösungsorientierte Fragen in den helfenden Gesprächsformaten Coaching und Psychotherapie aus linguistischer Sicht ${ }^{1}$. Für die Psychotherapie wurden Lösungsorientierte Fragen (LoF) ursprünglich definiert als initiierende sprachliche Interventionen auf Seiten der professionell Helfenden, mit denen Lösungen für im Gespräch thematisierte Probleme und Anliegen von Patient*innen gesucht sowie ihre diesbezüglichen Erwartungen und Wünsche an die persönliche und/oder berufliche Zukunft, aber auch die konkrete helfende Interaktion, ermittelt werden (Mack et al. 2016; SpranzFogasy et al. 2018; Kabatnik et al. 2019; Läpple et al. 2021).

Basierend auf gesprächsanalytischen Vorarbeiten wird die Interaktionstypspezifik von LoF im Coaching und in der Psychotherapie, vor allem im Hinblick auf ihr lokales Veränderungspotential, komparativ (re-)analysiert und dokumentiert. Dazu werden die formalen, funktionalen, kontextuellen und Coaching-spezifischen Charakteristika von LoF beschrieben und es wird aufgezeigt, wie sie zur lokalen Veränderung im Coaching-Gespräch bzw. zum Coaching-Prozess beitragen (vgl. Graf und Kabatnik im Druck). Parallel dazu werden diese Ergebnisse mit Erkenntnissen zu LoF aus dem Beratungsformat Psychotherapie bzw. genauer, aus OPD Diagnose-Gesprächen verglichen (Spranz-

\footnotetext{
${ }^{1}$ Die Vergleichbarkeit der vorliegenden Daten ist allerdings nur eingeschränkt gegeben und diese Einschränkung wird in der vorliegenden Argumentation stets kritisch mitbedacht: Bei den PsychotherapieDaten handelt es sich um Diagnose-Gespräche aus dem Bereich der Operationalisierten Psychodynamischen Diagnostik, während sich die Coaching-Daten auf ganze Prozesse des auf die intra-personale Ebene der Klient*innen fokussierenden Coaching-Ansatzes Emotional Intelligentes Coaching beziehen. Die hier generierten komparativen Erkenntnisse müssen daher in einem nächsten Schritt anhand weiterer Daten aus den Bereichen Psychotherapie und Coaching überprüft und gegebenenfalls adaptiert werden. Nichtsdestotrotz leistet die vorliegende Untersuchung einen wichtigen ersten Beitrag zum gesprächslinguistischen Verständnis von Lösungsorientierten Fragen als zentraler Intervention in Therapie und Coaching mit Fokus auf ihre Interaktionstyp-Spezifik und ihr jeweiliges Veränderungspotential.
}

Fogasy et al. 2018; Kabatnik et al. 2019; Läpple et al. 2021) und die Interaktionstyp-spezifischen Gemeinsamkeiten und Unterschiede vor dem Hintergrund der jeweiligen Handlungsrationale interpretiert. Eine erste Ausdifferenzierung des Fragetyps LoF für das Beratungsformat Coaching wird vorgeschlagen.

Unser Beitrag reiht sich ein in die gesprächslinguistische Vergleichsforschung zu helfenden Formaten bzw. zu sprachlichen Intervention (Weiste und Peräkylä 2013; Graf et al. 2014; Pick 2017; Günthner 2019; Graf, Scarvaglieri und Spranz-Fogasy (eds.) 2019) und in die vergleichende linguistische Forschung zu Psychotherapie und Coaching (Pawelczyk und Graf 2011; Graf und Pawelczyk 2014; Spranz-Fogasy et al. 2019a, b; Graf und Kabatnik im Druck). Das Herausarbeiten Format-spezifischer diskursiver Charakteristika ermöglicht ein fundierteres Verständnis ihrer professionsfokussierten Gemeinsamkeiten und Unterschiede auf der konkreten sprachlich-kommunikativen Handlungsebene. Da sich Coaching insgesamt, und der hier im Zentrum stehende Ansatz Emotional Intelligentes Coaching insbesondere, in seinem helfenden Handeln einerseits stark auf therapeutische Interventionen stützt (vgl. Crowe 2017, S. 86f., Deplazes 2016; Graf 2019), sich andererseits aber dezidiert und explizit von Therapie abgrenzt (Peltier 2010; Drath 2012; Cavanagh und Buckley 2014), ist der Vergleich dieser beiden Formate auf der Handlungsebene für eine fundierte Abgrenzungsdebatte unerlässlich.

Die vorliegenden (Re-)Analysen von LoF sind auch Teil der linguistischen Forschung zu transforming sequences bzw. Veränderung induzierende Sequenzen im therapeutischen Kontext (Voutilainen et al. 2011, 2018; Weiste und Peräyklä 2013; Voutilainen und Peräkylä 2016; Peräkylä 2019; Kabatnik et al. 2019) und im Coaching-Kontext (Spranz-Fogasy et al. 2019; Graf und Kabatnik im Druck; Kabatnik und Graf in Vorbereitung). Sie erweitern unsere Erkenntnisse darüber, wie einzelne diskursive Praktiken in ihrer sequenziellen und interaktionalen Organisation neues Wissen konstituieren und damit Veränderung als endemisches Ziel von Psychotherapie und Coaching ermöglichen. Während sich der aktuelle Beitrag dem Typ-inhärenten, aber auch dem lokalen ${ }^{2}$ Veränderungspotential von LoF in diesen beiden verwandten Formaten widmet, wird in einem weiteren Beitrag die longitudinale Perspektive (wie bei Voutilainen et al. 2011; Bercelli et al. 2013; Kabatnik et al. 2019) eingenommen, wodurch die durch LoF

\footnotetext{
${ }^{2}$ Vereinfacht wird das lokale Veränderungspotential im Kontext gelungener Sequenzen und das globale Veränderungspotential im Kontext der Zielerreichung definiert.
} 
evozierte, globale Veränderung entlang gesamter Therapieund Coaching-Sitzungen bzw. Prozesse vergleichend dokumentiert und interpretiert wird (vgl. Kabatnik und Graf in Vorbereitung).

Der erste Teil des vorliegenden Beitrags beschreibt Coaching und Psychotherapie aus linguistischer Perspektive und skizziert und kontrastiert ihre jeweilige Handlungsrationale im Hinblick auf Veränderung. Im Anschluss werden gesprächsanalytische Studien zu Fragen bzw. FrageAntwort-Reaktion Sequenzen als zentrale Intervention in helfenden Formaten zusammengefasst. Im zweiten Teil stehen aktuelle Ergebnisse zu LoF im Emotional Intelligentem Coaching (Graf und Kabatnik im Druck) und in den psychotherapeutischen Diagnosegesprächen (Kabatnik et al. 2019; Läpple et al. 2021) im Zentrum: Nach Beschreibung der diesen Studien zugrunde liegenden Datenbasis und Analysemethode werden die formalen, funktionalen und kontextuellen Charakteristika von LoF aufgeführt sowie ihre Interaktionstypspezifik in beiden Gesprächsformaten dargelegt. Auf dieser Basis erarbeiten wir einen kritischen Vergleich über Gemeinsamkeiten und Unterschiede dieser Frage-Praktik mit Bezug auf ihr jeweiliges lokales Veränderungspotential vor dem Hintergrund ihrer beraterischen Handlungsrationale und zeigen eine erste Erweiterung der Definition von LoF im Coaching auf. Der Beitrag schließt mit einem Ausblick auf nächste Analyseschritte zum globalen Veränderungspotential (Kabatnik und Graf in Vorbereitung).

\section{Coaching und Psychotherapie aus linguistischer Perspektive - same or different?}

Coaching und Psychotherapie stellen helfende Interaktionen dar (Miller und Considine 2009; Graf im Druck; Pick und Scarvaglieri im Druck), initiiert um ,,...) die Klient/ innen dabei zu unterstützen, zu begleiten und/oder ihnen zu helfen, ihre physische, psychische, intellektuelle und/ oder emotionale Verfassung zu verändern, zu stärken oder ihre Probleme im Zusammenhang damit zu lösen" (Graf und Spranz-Fogasy 2018b, S. 5). Dieser Fokus auf das helfende Handeln betont die Handlungsebene anstelle der externen professionellen Disziplin und rückt das Gespräch als primäres Medium und Methode in den Mittelpunkt der (analytischen) Aufmerksamkeit. Im Zentrum des Gesprächs stehen die übergeordneten kommunikativen Kernaufgaben ,Beziehungsgestaltung ' und ,Wissensgenerierung und Wissensvermittlung' (Spranz-Fogasy und Graf 2018b). Beratungsgespräche sind gekennzeichnet von ,grundlegenden Wissens- und Betroffenheitsasymmetrien“ (Spranz-Fogasy 2010, S. 74), d.h. die professionell agierende Person verfügt über Wissen, über das die Beratung und Unterstützung su- chende Person nicht verfügt, das aber für die zu behandelnde Fragestellung von Relevanz ist. Gleichzeitig sind Therapeut*in oder Coach von dem jeweiligen Thema - anders als Patient*innen/Klient*innen - nicht persönlich betroffen und haben somit eine für die Lösung des Problems (hilfreiche und notwendige) professionelle Außenperspektive. ,Beziehungsgestaltung ' und ,Wissensgenese und -vermittlung ' bestimmen die professionelle Agenda der helfenden Interaktionstypen und prägen darüber hinaus das konkrete Gespräch hinsichtlich des Ablaufs und des Inhalts auf der lokalen Mikroebene; der „quasi-conversational character“ der Interaktion (Drew und Heritage 1992; Marciniak et al. 2016) wird jedoch beibehalten.

Helfen in diesen Kontexten bedeutet ,sprachliches Helfen" (Pick und Scarvaglieri im Druck). Genauer definieren Pick und Scarvaglieri (im Druck) sprachliches Helfen als ein Abnehmen von (Teil-)Handlungen, wobei sich dieses Abnehmen nicht auf praktisches Handeln, sondern auf eine mentale Vorstrukturierung des Handelns bezieht. Während diese Handlungsstruktur in verschiedenen helfenden Handlungskonstellationen grundlegend dieselbe ist (Pick und Scarvaglieri im Druck, 2019), ist sie - je nach Einbettung des Helfens in unterschiedliche übergeordnete Handlungskomplexe (wie Coaching oder Psychotherapie) und ihre jeweils unterschiedlichen übergeordneten Zwecke (vereinfacht: Lösungsgenese vs. Problemverstehen) - als graduelles Phänomen mit stärkeren und schwächeren Ausprägungen zu verstehen, was sich in ihrer sprachlichen Realisierung im lokalen Hier-und-Jetzt der jeweiligen helfenden Gespräche manifestiert. Trotz der zugrunde liegenden Asymmetrie der Beteiligten im Hinblick auf Lösungskompetenz etc., wird das Helfen von allen Beteiligten gemeinsam prozedural und interaktional vollzogen. Auch die Hilfeempfangenden Personen sind also an der Interaktion beteiligt (Spranz-Fogasy und Graf 2018b), u.a. indem sie zunächst Hilfebedürftigkeit anzeigen (vgl. Marciniak 2017), die helfende Interaktion aktiv suchen und in Anspruch nehmen, aber auch ihre Anliegen schildern, Ziele klären und nach Lösungen suchen.

Sprachliches Helfen in beraterischen Kontexten wie Coaching und Therapie wird realisiert durch diskursive Praktiken, wie Interpretationen, (Re-)Formulierungen, Extensionen oder Fragen, sowie deren interaktive Weiterbearbeitung durch die Beteiligten entlang des ko-konstruierten HilfeGesprächs. Grundlegend für diese Betrachtung sprachlichen Helfens ist, dass der Einsatz der diskursiven Praktiken darauf abzielt, (lokale und globale) Veränderung zu induzieren. Hierbei orientieren sich die professionell Beratenden - im Sinne der appropriate responsiveness (Stiles 2013; Kramer und Stiles 2015) - sowohl am Gegenüber und der gemeinsamen lokalen in-situ Interaktion als auch an der zugrundeliegenden Handlungsrationale, die ihre professionelle Agenda bestimmt. Dass Coaching und Psycho- 
therapie als Format-endemische Ziele die Veränderung in unterschiedlichen privaten und/oder beruflichen Bereichen haben, wird an verschiedenen Stellen in der jeweiligen Praxis- und Forschungsliteratur hervorgehoben: So formulieren Whitworth et al. (1998, S. xix) für Coaching, ,(p)eople come to coaching for lots of different reasons, but the bottom line is change“. Und für Psychotherapie argumentiert Peräkylä (2013, S. 573), „change of some sort is the motivation for all psychotherapies" und elaboriert an anderer Stelle ,(i)t is characteristic for psychotherapy as interaction that it aims toward change in the feelings, thoughts, and behaviors in the client" (Peräkylä 2019, S. 258).

Während es auf institutioneller und professionssoziologischer Ebene sowie auf der Ebene der sozialen Zuschreibungen und Konnotationen bedeutende Unterschiede gibt (insbesondere wird stets die psychische Gesundheit der Coaching-Klient*innen betont (vgl. Drath 2012, S. 16; Cavanagh und Buckley 2014, S. 405)), bietet sich ein linguistischer Vergleich zwischen Coaching und Psychotherapie gerade wegen des beiden Formaten inhärenten Fokus auf Veränderungsgenese und der Bedeutung des Gesprächs zur Genese solcher Veränderung, an: Dazu werden im Coaching - dem deutlich jüngeren und weniger erforschten Format vorrangig Modelle und Interventionen verwendet, deren Ursprung in der Psychotherapie liegen (Drath 2012; Deplazes 2016; Graf 2019).

Um die für die Coaching-Praxis und die laufende Professionalisierung relevanten Unterschiede zwischen Coaching und Therapie herauszuarbeiten und sie so klarer voneinander abgrenzen zu können, bedarf es der Dokumentation und Analyse formaler, funktionaler, kontextueller und Interaktionstyp-spezifischer Charakteristika der diskursiven Praktiken und ihrer sequenziellen Einbettung in die konkreten in-situ Interaktionen zwischen Coach und Klient*in wie sie bereits für die Psychotherapie vorliegen (Peräkylä et al. 2008; Mack et al. 2016, S. $37 \mathrm{f}$.) Von besonderer Relevanz erscheint dabei eine (komparative) Erforschung von Fragepraktiken, da sie in der Coaching-Praxis als zentrale Intervention dargestellt werden, im Unterschied zur Psychotherapie aber kaum erforscht sind (vgl. Graf und Spranz-Fogasy 2018a; Graf et al. 2020; Graf und Kabatnik in Vorbereitung). Aufgrund der sowohl für Therapie als auch für Coaching zentralen Veränderungsfokussierung, d.h. dem Wunsch, Lösungen für die im Gespräch thematisierten Probleme in und durch das Gespräch selbst zu generieren, bieten sich LoF besonders für einen gesprächsanalytischen Vergleich an, um die theoretisch-handlungsrationalen Gemeinsamkeiten, aber auch Unterschiede, der beiden helfenden Formate auf der interaktiven Ebene beleuchten zu können. Um mit Mack et al. (2016, S. 82) zu sprechen: „Der sequenzlogische ,Auftrag ' zu antworten geht also bei lösungsorientierten Fragen Hand in Hand mit dem therapeutischen Auftrag, das mit der Frage gestellte
Problem zu lösen“. Vorab skizzieren wir dafür Fragen im Allgemeinen in helfenden bzw. therapeutischen Gesprächen aus gesprächsanalytischer Perspektive.

\section{Fragen in helfenden Gesprächen - „not an innocent thing to do" (Steensig und Drew 2008, S. 515)}

Fragen sind - im Unterschied zu den respondierenden sprachlichen Interventionen wie (Re-)Formulierungen, Interpretationen oder Extensionen - initiierend und fungieren grundsätzlich als Aufforderung zum Dialog. Fragen dienen „dem Wunsch nach Veränderung im Erleben, Handeln und Kommunizieren“ (Spranz-Fogasy et al. 2018, S. 112) und sind wichtige sprachlich-interaktive Bausteine zur Herstellung gemeinsamen Wissens (Deppermann 2015a), einem zentralen Zweck helfenden sprachlichen Handelns. Detaillierter formuliert Köller (2004, S. 662):

Fragen leiten hypothetische Vorstellungsprozesse ein, die eine immanente Tendenz haben, in Selbstreflexionsprozesse überzugehen. Fragen helfen uns, Wissensdefizite zu lokalisieren, Wissensbedürfnisse zu thematisieren, Interessen für Ursachen und Funktionen zu artikulieren, Bezüge zur Vergangenheit sowie Zukunft herzustellen [...]. Fragen setzen einerseits immer Erfahrungen voraus, sie sind andererseits aber auch dazu bestimmt, neue Erfahrungen zu ermöglichen, weil sie einen Wechsel von Sehepunkten und Wahrnehmungsperspektiven beinhalten.

Fragen regen dabei - initiiert durch professionell Agierende und ihr spezifisches professionelles Wissen - oftmals Reflexionsprozesse in Patient*innen/Klient*innen an, die laut Muntigl und Zabala (2008, S. 188) endemisch für Veränderung sind: „Adequate reflection on one's experience is often seen as a stepping stone to change because reflection can allow the client to construe his or her life and social relationships in additional and alternative ways". Fragen bilden hinsichtlich der angestrebten Intersubjektivitätsherstellung das Bindeglied zwischen „Verstehen“ und „Erfahren wollen“, da sie einerseits Wissensdefizite, aber auch Verstandenes und Bekanntes, dokumentieren, kommentieren, bewerten und präsupponieren; dabei setzen sie durch den spezifischen Adressat*innenzuschnitt ein bestimmtes Antwortformat konditionell relevant und geben durch die Formulierung bereits Aufschluss über gegebene thematische und inhaltliche Präferenzen. Fragen sind hierbei retro- und prospektiv relevant (Spranz-Fogasy et al. 2018, S. 115f.), d.h. sie beziehen sich auf das im Gespräch Vorherige und prägen das im Gespräch Folgende. Sie nehmen dabei ei- 
ne Sonderstellung in Bezug auf agency ${ }^{3}$ und epistemische Autorität der Teilnehmenden ein: So befindet sich der/die Fragende in agentiv dominanter Position gegenüber den Gesprächspartner*innen, die durch die konditionelle Relevanz mit einem Handlungsauftrag im Sinne des Lieferns einer Antwort konfrontiert werden. Fragen weisen demnach gesprächssteuernde Funktionen auf und können Dominanz, Kontrolle und Macht ausüben (Spranz-Fogasy et al. 2018, S. 117 f.; vgl. auch Tiittula 2001; Steensig und Drew 2008; Tracy und Robles 2009). Gleichzeitig können die Gefragten, trotz bzw. wegen des Prinzips der konditionellen Relevanz auf unterschiedliche Art und Weise reagieren bzw. respondieren (vgl. MacMartin 2008).

Für Psychotherapie-Gespräche konnten bis dato in der gesprächsanalytischen Forschung drei prototypische Funktionen bzw. Typen von Fragen hinsichtlich ihrer kontextuellen und sequenziellen Einbettung bzgl. formaler, funktionaler und Interaktionstyp-spezifischer Charakteristika identifiziert werden (für eine Zusammenfassung vgl. aktuell Spranz-Fogasy 2020): Fragen zur Aufklärung des Problemsachverhalts (prototypisch sind hierfür Beispielnachfragen, wie z.B. Haben Sie ein Beispiel dafür?) (Spranz-Fogasy 2019a, b), Fragen zur Erklärung und Ursachenerforschung (wie etwa Fragen zur Kollaborativen Erklärungsfindung, wie z.B. Woher kommt das?) und Fragen zur Lösungsfindung (wie etwa Lösungsorientierte Fragen, wie z.B. Was würden Sie sich da wünschen?). Diese Psychotherapie-basierte Fragentypologie dient der gesprächslinguistischen Erforschung von Fragepraktiken im Coaching als Orientierung im Sinne von senitizing concepts (Blumer 1954). Dabei offenbaren erste Analysen u. a. die Notwendigkeit, etablierte Fragetypen, wie Lösungsorientierte Fragen, und ihre Funktion stärker zu differenzieren (siehe unten).

\section{Daten und Methode}

Grundlage des vorliegenden Vergleichs bilden die gesprächsanalytischen Vorarbeiten bzw. Ergebnisse zu LoF

\footnotetext{
3 Während sich das Konzept ,agency“ in vielen verschiedenen Disziplinen findet, unterliegt unserer Argumentation ein gesprächslinguistisch-interaktionales Verständnis von agency. Laut Marciniak (2017, S. 18) werden ,(...) Überzeugungen über die Wirkmächtigkeit eigener und fremder Handlungen sowie die Verteilung der Handlungsmacht auf die Teilnehmer einer sozialen Situation [...] über Sprache in der Interaktion beobachtbar und beschreibbar". Hierbei kommt die Handlungsrationale der hier im Fokus stehenden helfenden Interaktionen Coaching und Psychotherapie bezüglich der Vorannahmen und Zuschreibung von Wirkmächtigkeit und Handlungsfähigkeit der Klient*innen bzw. Patient*innen auf der Ebene des Gesprächs zum Tragen (für eine tiefer gehende Diskussion des Begriffs agency aus gesprächslinguistischer Sicht siehe auch Lucius-Hoehne (2012), Deppermann (2015b) und Kook (2015)).
}

Fragen in psychotherapeutischen Gesprächen von SpranzFogasy et al. (2018) und Kabatnik et al. (2019) sowie von Graf und Kabatnik (im Druck) zu LoF in Coaching-Gesprächen. Das Korpus zu LoF in der Psychotherapie umfasst 15 psychotherapeutische Diagnosegespräche, die im Jahr 2009 audio- und videotechnisch aufgezeichnet wurden und einer Forschungskooperation zwischen dem Leibniz-Institut für Deutsche Sprache in Mannheim und der Universitätsklinik für Allgemeine Innere Medizin und Psychosomatik in Heidelberg entstammen (vgl. Spranz-Fogasy et al. 2018). Bei den Gesprächen handelt es sich um Erstgespräche, die mit dem Konzept und Manual der Operationalisierten Psychodynamischen Diagnostik (OPD; Arbeitskreis OPD 2014) zu Forschungszwecken des OPD-Therapiekonzepts geführt wurden, wobei eine weitere Behandlung der Patient*innen durch die jeweiligen Therapeut*innen nicht vorgesehen war. Die psychotherapeutischen Diagnosegespräche wurden von 5 Therapeut*innen mit 15 Patient*innen geführt, sind im Durchschnitt 75 min lang und haben eine Gesamtlänge von ca. $19 \mathrm{~h}$. In 12 der $15^{4}$ psychotherapeutischen Gespräche wurden insgesamt 27 LoF lokalisiert.

Das Graf und Kabatnik (im Druck) zugrundeliegende Coaching-Korpus umfasst 3 komplette Coaching-Prozesse (insgesamt 12 Gespräche) aus dem Bereich des Führungskräfte-Coaching und entstammt einem größeren Korpus zu Emotional Intelligentem Coaching (vgl. Graf 2019), aufgezeichnet zwischen 2007 bis 2009 von den Coaches. Die für die Analyse von LoF daraus ausgewählten Coaching-Prozesse enthalten 4,5 bzw. 3 Sitzungen von 1,5 bis $2 \mathrm{~h}$ und entsprechen mit insgesamt ca. $20 \mathrm{~h}$ ungefähr der psychotherapeutischen Datenmenge. In den 12 Coaching-Gesprächen konnten insgesamt $105 \mathrm{LoF}$ identifiziert werden (Graf und Kabatnik im Druck).

Einschränkend für die Aussagekraft des vorliegenden Vergleichs muss an dieser Stelle nochmals darauf hingewiesen werden, dass es sich bei den Psychotherapie-Daten um Diagnose-Gespräche aus dem Bereich des OPD handelt, bei denen es zunächst primär um die Erstellung einer Diagnose (als Basis für eine darauf aufbauende, weiterführende Therapie) und nicht um die Er- und Bearbeitung von Lösungen geht. Allerdings handelt es sich bei den Coaching-Daten auch nicht um klassisch systemisch-lösungsorientiertes Coaching (Seiger und Künzli 2012; Middendorf und Salomon 2017), sondern um einen auf die intra-personale, emotionale Ebene der Klient*innen fokussierender Coaching-Ansatz (Graf 2019), der stark therapeutische Diskurselemente enthält (Schulz 2015; Graf 2019). Den therapeutischen Diagnose-Gesprächen werden als Coaching-Daten ganze Coaching-Prozesse, und nicht nur Erst-

\footnotetext{
${ }^{4}$ In drei der Gespräche fanden sich keine LoF. Lösungen wurden entweder auf eine andere Weise oder aufgrund der Sensibilität der Thematik gar nicht verhandelt.
} 
gespräche, gegenübergestellt, da so die jeweiligen Interaktionsformate - in sich abgeschlossen - verglichen werden können. Die hier generierten komparativen Erkenntnisse dieser beiden speziellen Ausprägungen therapeutischer und Coaching-Daten müssen allerdings in einem nächsten Schritt anhand repräsentativerer Daten aus den Bereichen Psychotherapie und Coaching überprüft und gegebenenfalls adaptiert werden. Die Therapie- und Coaching-Daten waren jeweils gesprächsanalytisch im Sinne von Deppermann (2008) und Brinker und Sager (2010) analysiert worden: Die Gesprächsanalyse nimmt die sequenzielle Organisation von Gesprächen im Kontext von aufeinander aufbauenden und sich gegenseitig bedingenden Äußerungen bzw. Beiträgen (=turns) der Gesprächsteilnehmer*innen als Motor und Vehikel der Interaktion in den analytischen Blick (Schegloff 2007; Deppermann 2018). Wie eine Äußerung von Sprecher*in A verstanden wird, zeigt Sprecher*in B in ihrer Reaktion darauf an und Sprecher*in A signalisiert im nächsten Schritt, der 3. Position, ob sie sich richtig verstanden fühlt. Und auch die erste Äußerung ist bereits oder kann schon als Reaktion auf das betrachtet werden, was zuvor (wie) im Gespräch gesagt wurde. Fragen als Fokusäußerung (target actions, Peräkylä 2019) in professionellen Hilfe-Gesprächen sind ein Musterfall der sequenziellen Organisation von Gesprächen: Stärker als andere sprachliche Handlungen erzeugen Fragen einen Reaktionszwang für Gesprächspartner*innen, wobei Veränderungsarbeit somit geradezu ,provoziert" wird. Anstellte einer isolierten Betrachtung von Fragen werden deshalb in der Gesprächsanalyse „FrageAntwort“ bzw. „Frage-Antwort-Reaktion Sequenzen“ analysiert (Graf et al. 2020).

\section{Lösungsorientierte Fragen in Coaching und Psychotherapie: Ein Vergleich}

${ }^{\circ} \mathrm{h}($.$) wenn sie ähm (0.68)$ drei wünsche frei hätten (1.03) ((schmatzt)) abgesehen (0.52) von ihren eigenen beschwerden (.) ${ }^{\circ} \mathrm{h}(0.28)$ was würden sie sich wünschen (1.6)

(Beispiel aus dem Psychotherapie-Korpus: T 3-1, 01:25:12-01:25:20)

Aber $((1,1 \mathrm{~s}))$ Was Sie beschäftigt ist: ,Was kann ich da noch tun, um s/ ja das, das, das, das/ die Gesprä-

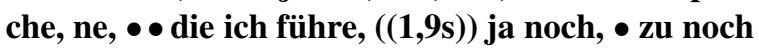
besseren Ergebnissen führen.“..••

(Beispiel aus dem Coaching-Korpus: KL2_1-2)

\footnotetext{
${ }^{5}$ Für die Anonymisierung der Gesprächsbeteiligten wurden Abkürzungen verwendet. Die Grapheme stehen für den jeweiligen CoachingProzess, z. B. KL1, die Zahlen drücken die Nummerierung zur Sitzung aus, z. B. 2, also die zweite Sitzung im Coaching-Prozess KL1.
}

Mack et al. (2016) haben Lösungsorientierte Fragen erstmals als eigenständigen Fragetyp mit endemischen funktionalen Eigenschaften im psychotherapeutischen Gespräch identifiziert: LoF etablieren den Handlungsauftrag einer Lösungsfindung für ein zuvor im Gespräch ausgehandeltes Problem bzw. komplex miteinander zusammenhängende Probleme der Patient*innen (Mack et al. 2016, S. 81). Durch LoF initiieren Therapeut*innen eine Refokussierung von Problemexploration auf eine zukunftsgerichtete Perspektive hin, mittels derer Patient*innen auf potenziell mögliche Lösungen für ihre Probleme aufmerksam gemacht werden sollen (vgl. Läpple et al. 2021, S. 38). Patient*innen werden dabei eingeladen, in einem durch den projektiven und prospektiven Charakter von LoF geschützten Rahmen eigenständig Lösungen für ihre Probleme zu entwickeln sowie Wünsche und Hoffnungen zu formulieren. Die in der Therapie thematisierten Probleme beziehen sich dabei stets auf eine mehr oder weniger umfassende eingeschränkte Handlungsfähigkeit der Patient*innen i. S. reduzierter Agency (Deppermann 2015b; Kook 2015; Marciniak 2017).

Lösungsorientierte Fragen im Coaching wurden von Graf und Kabatnik (im Druck), aufbauend auf den Erkenntnissen aus der linguistischen Psychotherapieforschung, zum ersten Mal in den gesprächsanalytischen Blick genommen. In Adaption an die Definition von Kabatnik et al. (2019) und Läpple et al. (2021) für das Gesprächsformat „Psychotherapie" handelt es sich um eine prospektive Aufforderung des*der Coach an Klient*innen, Lösungen für die im Coaching-Gespräch thematisierten Probleme oder Anliegen, sowie konkrete Maßnahmen und Strategien zu entwickeln, bereits vorhandene Lösungsressourcen $\mathrm{zu}$ aktivieren und handhabbar zu machen, aber auch ihre Erwartungen an die (berufliche) Zukunft und/oder an das Coaching zu formulieren (vgl. Graf und Kabatnik im Druck). Im Coaching findet sich von Beginn eine starke Fokussierung auf Lösungsfindung/-orientierung und die meisten Handlungen von Coaches stehen im Dienste dieses übergeordneten Ziels. Es geht nicht darum, die Handlungsfähigkeit bzw. agency generell wieder herzustellen, sondern eine mangelnde Fähigkeit zur Handlungsentscheidung auszuloten und einen Handlungswiderstand zu überwinden (Läpple et al. 2021). Dabei wird grundsätzlich ein handlungsfähiges Gegenüber als Klient*in angenommen, das bereits zu Beginn des Prozesses über Lösungsfindungskompetenz verfügt.

Im Durchschnitt finden sich pro Stunde 8,9 LoF im Coaching-Gespräch, im Therapie-Gespräch dagegen sind es nur 2,25 LoF (siehe unten). Die starke Fokussierung auf Lösungsfindung und -orientierung im Coaching lässt sich also bereits quantitativ in den untersuchten Daten nachweisen. 


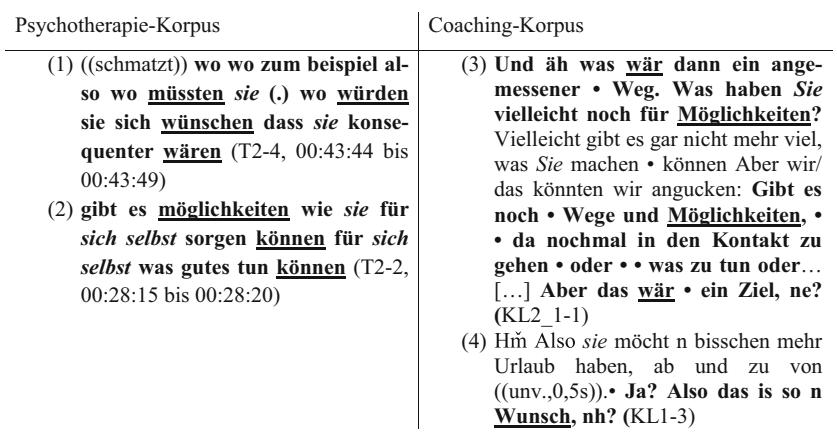

Abb. 1

\subsection{Formale Eigenschaften von LoF im Vergleich}

Bezüglich des Formulierungsdesigns weisen LoF sowohl in der Psychotherapie $(n=20,74 \%)$ als auch im Coaching $(n=84$, rund $80 \%)$ der Fragen das W-Frageformat auf (s. Bsp. 1 \& 3). V1-Fragen sind ebenfalls in beiden Gesprächsformaten möglich (Psychotherapie: $n=7,26 \%$, Coaching: $n=21,20 \%$ ), enthalten aber zumeist integrierte W-Fragen (s. Bsp. 2). LoF als Deklarativsatzfragen wurden lediglich im Coaching identifiziert ( $n=4,4 \%$, s. Bsp. 4), vgl. die folgenden Beispiele (Abb. 1).

Die Beispiele zeigen eine starke prospektiv-projektive Ausrichtung mit Fokus auf Vorstellungen, Wünsche, Fähigkeiten und Möglichkeiten in Bezug auf das Leben oder die Therapie selbst, die für LoF in der Psychotherapie und im Coaching charakteristisch sind. Die charakteristische Verwendung von Wahrnehmungs-, Psycho-, Modalund volitionalen Verben und Substantiven in beiden Gesprächsformaten (s. Bsp. 1-4) sowie der häufige Gebrauch des Konjunktivs mit Gegenwarts- oder Zukunftsbezug (im Psychotherapie-Korpus: Konjunktiv: $n=16$, 59\%, Zukunft: $n=3,11 \%$, Gegenwart $n=4,15 \%$, Zukunft und Gegenwart: $n=20,74 \%$; in Coaching-Daten: Konjunktiv: $n=57$, $54 \%$, Zukunft: $n=61$, rund $58 \%$, Gegenwart: $n=15$, rund $14 \%$, Zukunft und Gegenwart: $n=29,28 \%$ ) formulieren die geäußerten Inhalte als höchst tentativ und verhandelbar. Dies spiegelt sich auch auf semantisch-lexikalischer Ebene wider: In beiden Korpora werden Lösungsmöglichkeiten lexematisch explizit gemacht, wie z.B. Ziel, Wunsch oder Möglichkeit. Durch diesen hypothetischen Charakter der durch LoF thematisierten Inhalte, die ,auf die Zukunft bezogen und erst noch zu realisieren sind" (Mack et al. 2016, S. 81), müssen Patient*innen und Klient*innen keine Konsequenzen für die von ihnen geäußerten Lösungsmöglichkeiten fürchten, sondern können ,,in einem geschützten Rahmen gedanklich wie sprachlich mit verschiedenen Szenarien und eben auch Lösungsmöglichkeiten ,experimentier[en]“" (Mack et al. 2016, S. 81). LoF können dabei sowohl kognitive als auch emotiv-volitionale Projektionen zulassen.

\begin{tabular}{|c|c|}
\hline Psychotherapie-Korpus & Coaching-Korpus \\
\hline $\begin{array}{l}\text { (5) (.) sagen wir mal so motivations } \\
\text { ((atmet ein; ca. } 1,19 \mathrm{~s})) \text { löcher }(.) \text { sind } \\
\text { ja }(.)(\mathrm{n}) \text { was was }(1.01) \text { vielen men- } \\
\text { schen (.) passiert im (.) äh studium } \\
\text { oder sonst wie und dann gibt_s ja } \\
{ }^{\circ} \text { hh für die meisten }(0.49) \text { irgenwie } \\
\text { dann so_n ziel so_n langfristiges ziel } \\
\text { was dann }(0.35) \text { ihnen dazu (.) hilft } \\
(0.23) \text { durchzuhalten oder so ((atmet } \\
\text { ein; ca. } 1,43 \mathrm{~s})) \text { sowas }(.) \text { bei } \text { ihnen } \\
\text { irgend ne idee was sie wo sie gerne } \\
\text { hin möchten T1-4 (00:43:31 bis }\end{array}$ & $\begin{array}{l}\text { (6) }((1,5 \mathrm{~s})) \text { Blick auf die Uhr. Wir haben } \\
\text { also jetzt noch • ne halbe Stunde, ja? } \\
\text { - Was wär denn, sagen wir, bezo- } \\
\text { gen auch auf diie, } \cdots \text { die (Ziele) } \\
\text { was wär heute noch wichtig zu be- } \\
\text { sprechen? }((2,3 \mathrm{~s})) \text { Erstmal ist so die } \\
\text { Frage: Haben Sie das Gefühl, das } \\
\text { sind die zwei wichtigsten Elemente, } \\
\text { die wir bis jetzt besprochen haben? } \\
\left(\mathrm{KL} 2 \_1-3\right)\end{array}$ \\
\hline
\end{tabular}

Abb. 2

Auffällige zögerliche Formulierungsdynamik in fast $3 / 4$ aller LoF im Psychotherapie-Korpus $(n=19,70,4 \%)$ und rund $93 \%$ im Coaching-Korpus $(n=98)$ zeugt davon, dass dieses interaktive Unterfangen sowohl für Therapeut*innen bzw. Coaches als auch Patient*innen und Klient*innen relativ herausfordernd zu sein scheint (Spranz-Fogasy et al. 2018, S. 122), vgl. die folgenden Beispiele (Abb. 2).

Durch die auffällige zögerliche Formulierungsdynamik in beiden Interaktionstypen können auch die Sensitivität der angesprochenen Themen und der aufgebaute Druck auf die Klient*innen, auf emotional-kognitiver Ebene innerhalb eines mehr oder weniger vorgegebenen zeitlichen Rahmens eine Lösung zu liefern, angezeigt werden. Eine weitere Hypothese ist, dass die sehr schnelle Fokussierung auf Lösungen im Coaching, ohne (ausreichende) Problemwertschätzung, zu dieser Zögerlichkeit führt. Weiter können LoF je nach Erfordernissen zur Kontextualisierung in beiden Gesprächsformaten unterschiedliche Komplexitätsgrade aufweisen, die von kurzen initiativen Äußerungen (s. Bsp. 2 \& 4; im Psychotherapie-Korpus: $n=6,22 \%$; im CoachingKorpus: $n=26$; rund $25 \%$ ) bis hin $\mathrm{zu}$ immer komplexer werdenden Äußerungen mit insertierten und/oder nachgeschobenen Klärungen von Rahmenbedingungen reichen (s. Beispiel 5 \& 6).

Auffallend ist in fast allen Fragen die explizite und $d i$ rekte Adressierung der Patient*innen und Klient*innen (im Psychotherapie-Korpus: $n=27,100 \%$; im Coaching-Korpus: $n=90,86 \%$ ), die teilweise mehrfach realisiert wird - trotz des dyadischen Settings (s. Bsp. 1-6; Kabatnik et al. 2019, S. 157). Diese kommunikative Praktik stellt zum einen die Patient*innen und Klient*innen sowie deren Ziele, Pläne und Wünsche in den Fokus des Gesprächs und leistet so implizite Beziehungsarbeit (vgl. Günthner im Druck). Zum anderen wird auf diese Weise die Eigenständigkeit der Klient*innen betont, da diese nun gefragt sind, eigene, introspektive Lösungsmöglichkeiten $\mathrm{zu}$ formulieren - im Kontrast zu Vorschlägen, die zum Beispiel familiär oder gesellschaftlich auferlegt sind. Auf diese Weise wird auch die Verantwortung für die zu formulierenden Lösungsmöglichkeiten auf die Klient*innen übertragen (vgl. 
(7) ist nur ne Frage so: wann, ne. Und äh wenn ich $\underline{\text { Sie jetzt frage, bezogen auf dieses, }}$

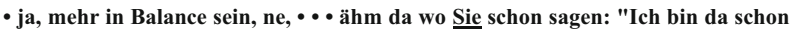
zufriedener.", ((1.1s)) was ist es, was $\underline{\text { Sie }} \mathbf{d a}, \mathbf{h} \overline{\mathbf{m}}$ sagen wir mal so, erreichen wol-

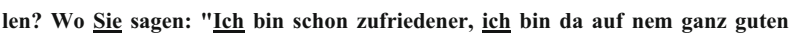

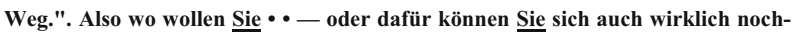
mal Zeit lassen - wo wollen $\underline{\text { Sie, }}$ ((1s)) ja, in nem halben Jahr, oder in nem • Jahr sein? (KL2 1-2)

Abb. 3

für Psychotherapie Kabatnik et al. 2019, S. 159). Betrachtet man die direkte und mehrfache Adressierung im Kontext reduzierter Agency im Vorlauf, wird Patient*innen und Klient*innen bereits in und durch die Frage ein handlungsfähiges Selbstbild entgegengesetzt, die Frage an sich suggeriert demzufolge schon Veränderung in den Patient*innen. Mithilfe dieser eindringlichen Adressierung wird nicht nur das Erfragen sehr persönlicher Inhalte und die Suche nach tatsächlichen Lösungen, sowie die Übertragung von Verantwortung auf Patient*innen wie Klient*innen ermöglicht, sondern auch die anschließende Beobachtung des Umgangs damit wie auch die Bereitschaft zu einer stärkeren Agency. Die stärkere Ausprägung dieses Phänomens in den Coaching-Daten ist dabei sicherlich motiviert von einer anzunehmenden bzw. zugeschriebenen durchschnittlich höheren Handlungsfähigkeit der Klient*innen im Coaching als in der Psychotherapie.

Im Unterschied zum Gesprächsformat der Psychotherapie wird die direkte Adressierung in den Coaching-Daten aber auf unterschiedliche Weise realisiert. Dies zeigt sich zum einen in der Verwendung von Sie vs. $D u$, wodurch die (In)Offizialität vergrößert bzw. verringert sowie Nähe oder Distanz zwischen den Interaktant*innen markiert wird und der „quasi-conversational character“ (Drew und Heritage 1992) von Coaching zum Ausdruck kommt. An zahlreichen Stellen in den Coaching-Daten findet sich darüber hinaus die zitierte Rede (Und • jetz is ja das ne Situation, wo Sie sagen: „Ich würd mir da wünschen, mich anders zu fühlen und mich anders zu verhalten. "IIs das richtig? (KL_1-2)). Die Adressierung wird im Ich-Format realisiert (vgl. Plank 1986, S. 286), was die Unmittelbarkeit der Proposition der Frage bereitstellt und sich durch diese Perspektivierung direkt auf die Klient*innen übertragen lässt.

Darüber hinaus konnte eine weitere Form der Adressierung im Coaching identifiziert werden, in der die Adressierung durch $D u$ realisiert wird: hier wird jedoch nicht die Klient*in selbst, sondern einer ihrer Persönlichkeitsanteile angesprochen (Was (wünscht si) sich der Teil von dir? - Also was/ wie könntest du dem Teil helfen? Auch die nächsten Wochen. ((4s)) Wenn der sich meldet, ((1s)) und - du spüren kannst, was bräuchte der Teil dann von dir. (KL_2)). Dadurch können unbewusste Bedürfnisse, Wünsche und Möglichkeiten priorisiert werden bzw. das Anliegen kann auf einer intra-personalen Ebene bearbeitet wer- den. Die Befragung von Persönlichkeitsanteilen in LoF im Coaching wird in rund $11 \%(n=11)$ realisiert, wodurch nicht nur die bewussten Wünsche und Möglichkeiten der Klienten*innen in den Fokus der Frage gerückt, sondern auch solche, die eher im Unbewussten angesiedelt sind und mithilfe der Lösungsorientierten Fragen als Ressource ins Bewusste überführt werden können. Deutlich wird hier die Herangehensweise des fokussierten Coaching-Ansatzes, Emotional Intelligentes Coaching, dessen Basis ein (therapeutischer) Ansatz basierend auf der Idee, dass die Persönlichkeit der Klient*innen aus unterschiedlichen Persönlichkeitsanteilen besteht, bildet. Diese können konträre Auffassungen vertreten, situationsabhängig mehr oder weniger stark im Vordergrund stehen und dabei häufig als Ursache der externen Anliegen der Klient*innen ausgemacht werden (vgl. Graf 2019).

Auffällig in den LoF im Coaching im Vergleich mit Psychotherapie ist, dass sie in den Coaching-Daten häufig, d.h. in rund $45 \%(n=47)$, innerhalb aufeinanderfolgender Frageketten realisiert werden. Innerhalb dieser Frageketten kann es zu zeitlichen, modalen und/oder thematischen Fokusverlagerungen kommen, wodurch ein Wechsel beispielsweise von einer retrospektiven zu einer prospektiven Betrachtung von problematischen Inhalten und der Hinwendung zu Lösungsmöglichkeiten angezeigt werden kann. Voran- und nachgestellte Fragen nehmen dabei Bezug auf dasselbe Thema (Abb. 3).

In Hinblick auf die zeitliche Dimension referiert die Frage in Beispiel 7 im Vorlauf der LoF auf die Gegenwart und Zukunft, wodurch der zeitliche Fokus in der Fragekette von der Gegenwart in Richtung Zukunft verlagert wird. Im Coaching-Gespräch initiieren Coaches also durch LoF mitunter eine Refokussierung eines gegenwärtigen Zustandes auf eine zukunftsgerichtete Perspektive hin, mittels derer Klient*innen auf mögliche Problemlösungen aufmerksam gemacht werden sollen.

In Bezug auf die Zeitlichkeit der Lösungsorientierten Fragen ist weiter auffällig, dass - im Unterschied zu den Psychotherapie-Daten - explizite zeitliche Begrenzungen oder Fixierungen verbalisiert werden, wie z. B. in (7) wo wollen Sie, ((1s)) ja, in nem halben Jahr, oder in nem • Jahr sein? (KL2 1-2). Ein Zeitraum für die antizipierte Veränderung wird so bereits sprachlich-kommunikativ bestimmt und festgelegt und dabei für die Klient*innen konkreter greifbar und verbindlicher. Dies manifestiert - auch auf gesprächslokaler Ebene - den Wunsch nach zeitlich determinierter und damit auch messbarer Veränderung im Coaching als handlungsleitende Maxime, aber auch klient*innenseitige Erwartung (siehe Graf und Wastian 2014 zu SMARTen Zielen im Coaching). 


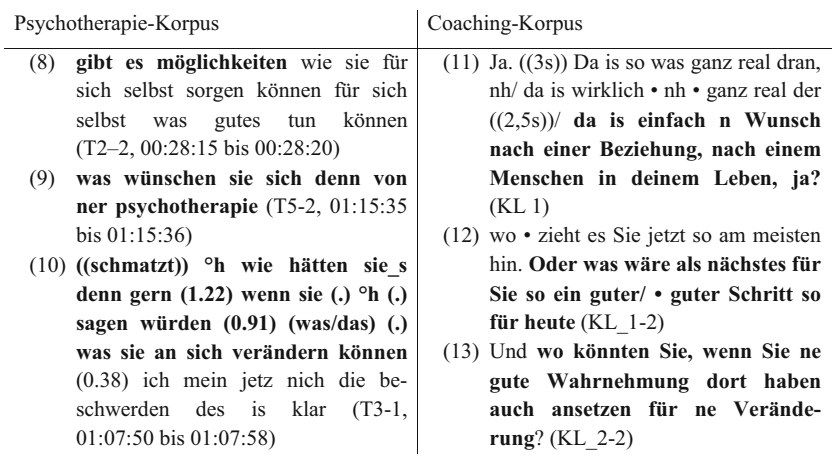

Abb. 4

\subsection{Thematische Gestaltung von LoF im Vergleich}

LoF beziehen sich in den therapeutischen, und auch den Coaching-Gesprächen thematisch häufig auf den beruflichen und/oder privaten Lebensbereich von Patient*innen und Klient*innen oder die aktuelle therapeutische Behandlung (vgl. Bröcher 2017, S. 35; Oelschläger 2017, S. 38) bzw. die Coaching-Sitzung. Dabei werden zumeist deren Wünsche, Hoffnungen, Vorstellungen oder Ziele außerhalb, aber auch innerhalb der Therapie bzw. des Coachings, aber auch ganz konkret und explizit die Veränderung (s. Bsp. 10 und 13) adressiert sowie (bestehende) Ressourcen aktiviert (Abb. 4).

Darüber hinaus beziehen sich LoF im Coaching häufig auch metakommunikativ beispielsweise auf die weitere Vorgehensweise bzgl. der thematischen Ausrichtung im Coaching selbst (s. Bsp. 12) ${ }^{6}$ : Aus interaktionaler Sicht zeigt sich insbesondere bei Letzterem das Verständnis von Coaching als Gespräch auf Augenhöhe (Jautz 2017), das den Beteiligten mit ihren verschiedenen Wissensbeständen Einfluss auf die (thematische) Gestaltung der Sitzung einräumt und nicht dem*der Coach die alleinige Verantwortung (aber auch Macht) für die inhaltliche Gestaltung und damit Problemlösung überträgt. Nicht nur bei Führungskräften als Klient*innen ist diese Zuschreibung von Handlungsfähigkeit und Eigenverantwortung (z.B. ähm, - wo würden Sie so als erstes Mal was Neues vielleicht auch ausprobieren wollen (KL_1-2)) auch zentral für die Akzeptanz und den Erfolg von Coaching.

Patient*innen dürfen in der Psychotherapie zwar auch ihre Wünsche äußern, s. Bsp. (9) bezüglich der Ziele der Psychotherapie im Allgemeinen. Die Formulierung sich von der Psychotherapie etwas wünschen zeigt hier jedoch an,

\footnotetext{
${ }^{6}$ An diesem Beispiel kündigt sich bereits an, dass für den Interaktionstyp Coaching Lösungsorientierte Fragen eher als Überbegriff für mehrere Fragetypen mit unterschiedlichen (genauer ausdifferenzierten) Funktionen zu verstehen sind; geht es nicht mehr um die Lösung des Problems, sondern um eine (kollaborative und explizite) Klärung nächster Schritte im Coaching, schlagen Dionne et al. (in Vorbereitung) vor, von „Agenda-Thematisierungsfragen“ zu sprechen.
}

\begin{tabular}{|c|c|}
\hline Psychotherapie-Korpus & Coaching-Korpus \\
\hline $\begin{array}{l}\text { (14) }\left(\left(\text { schmatzt)) }{ }^{\circ} \mathbf{h} \text { wie hätten sie_s }\right.\right. \\
\text { denn gern (1.22) wenn sie }(.){ }^{\circ} \mathbf{h}(.) \\
\text { sagen würden }(0.91) \text { (was/das) }(.) \\
\text { was sie an sich verändern können } \\
(0.38) \text { ich mein jetz nich die be- } \\
\text { schwerden des is klar (T3-1, } \\
01: 07: 50 \text { bis } 01: 07: 58)\end{array}$ & $\begin{array}{l}\text { (15) Und da wär die Frage: Wie können } \\
\text { Sie das noch mehr beeinflussen? } \\
\text { Also gibt es da Dinge, }((1,3 s)) \text { die } \\
\text { Sie noch nicht ausprobiert haben? } \\
((1,2 \mathrm{~s}))[\ldots]\left(\mathrm{KL} \_2\right)\end{array}$ \\
\hline
\end{tabular}

Abb. 5

dass es sich um ein stärker institutionalisiertes Gesprächsformat handelt (vgl. Marciniak et al. 2016, S. 2), in dem zwar Schwerpunkte gesetzt werden können, der Ablauf und die Leitung des Gesprächs jedoch dem*der Therapeuten*in unterliegt.

\subsection{Funktionale Eigenschaften von LoF im Vergleich}

Basierend auf den Analysen zu Lösungsorientierten Fragen in den therapeutischen Diagnosegesprächen liegt dort ihre primäre Funktion in der „Überprüfung von Bewusstseinsständen und Perspektiven des Patienten [sic] sowie dessen Fähigkeit zur Lösungsfindung" (Oelschläger 2017, S. 90f.). Dadurch können Therapeut*innen die psychische Verfassung der Patient*innen besser einschätzen und diagnostisch wertvolle Informationen gewinnen. Auch kann durch LoF implizit Kritik an bestimmten ,problematischen Haltungen, Verhaltensweisen oder Äußerungen des Patienten [sic]“ (ebd., S. 90) geübt und ihnen so eigene Unzulänglichkeiten aufgezeigt werden. Diese können dann im Gespräch vertieft behandelt werden. Weitere mögliche Funktionen sind die Ermittlung realer Möglichkeiten durch die Thematisierung konkreter Lösungsansätze in Bezug auf Wünsche, Ziele oder Lebenspläne der Patient*innen oder die Übertragung von Verantwortung in Bezug auf eine eigenständige sowie eigenverantwortliche Lösungsformulierung (vgl. Bröcher 2017, S. 37 f.). Dabei weisen LoF laut Bröcher (2017, S. 76f.) und Oelschläger (2017, S. 91) in den Psychotherapie-Daten nicht nur eine, sondern mehrere funktionale Eigenschaften gleichzeitig auf (Abb. 5).

Beispiel (14) geht die Erklärung des Therapeuten über schädliche Verhaltensweisen des Patienten voraus. Die darauffolgende LoF (14), mit der Veränderungswünsche abgefragt werden, zielt neben der Aushandlung einer tatsächlichen Lösung für das Problem auch auf den Ausdruck von Kritik über als veränderungswürdig eingestufte Verhaltensmuster sowie der Überprüfung, ob die zuvor formulierte Haltung des Therapeuten angenommen oder abgelehnt wird.

Ähnliche Funktionen können auch im Coaching-Korpus identifiziert werden. Auch dort kommt die Funktion von LoF zum Tragen, nämlich im diagnostischen Sinne den Bewusstseinszustand der Klient*innen und ihre Perspektiven und Lösungsfindungskompetenzen einzuschätzen: Coaches 
Abb. 6 Funktionale Differenzierung von Lösungsfindung/ -orientierung im Kontext von LoF

\begin{tabular}{|c|c|}
\hline pp & eispiel \\
\hline $\begin{array}{l}\text { (Vorhandene) Lösungsressourcen aufdecken - es sollen } \\
\text { bereits vorhandene Ressourcen der Klient*innen aufge- } \\
\text { deckt / an die Oberfläche gebracht / erfragt werden. Es } \\
\text { werden u.a. (bereits erkennbare) Lösungsmöglichkeiten } \\
\text { ausgetestet, Lösungswünsche und Vorstellungen formu- } \\
\text { liert. Oft stehen konkrete Handlungen im Vordergrund, um } \\
\text { Ressourcen aufzudecken. Fragen, die dieser Funktion } \\
\text { zugeordnet werden können, treten schon zu Beginn von } \\
\text { Sitzungen oder Prozessen auf und haben somit sowohl } \\
\text { aufklärende als auch lösungsorientierte Funktion. }\end{array}$ & $\begin{array}{l}\text { (16) Nehmen wir auch } \cdots \text { / und (spür nochmal), } \\
\text { nehmen wir mal an, du merkst, n Teil dieser • } \\
\text { Entwicklung, dieser Veränderung }((3,5 \mathrm{~s})) \text { is auch } \\
\text { mit diesem } • \text { Gefühl, anders umgehen zu kön- } \\
\text { nen. }((1,5 \mathrm{~s}))(\mathrm{Da} \text { is) "Hoppla, da }((1,5 \mathrm{~s})) \text { passiert } \\
\text { irgendwas, da kommt diese Anspannung", ((4s)) } \\
\text { und du merkst: "He, ich kann damit anders um- } \\
\text { gehen." ((1s)) Wie is das? Was machst du da? } \\
\text { ((2,5s)) Was machst du in dem Moment, wo du } \\
\text { merkst: "Und jetzt bin ich da in dieser Kamp- } \\
\text { fesstimmung."? ((1,5s)) In der Anspannung. • } \\
\text { • Wie gehst du dann damit um? (KL_1-2) }\end{array}$ \\
\hline $\begin{array}{l}\text { (Fehlende) Lösungsressourcen be-/erarbeiten - hier } \\
\text { werden einerseits aufgedeckte / bereits vorhandene Lö- } \\
\text { sungsressourcen aktiviert und konkretisiert, d.h. weiterbe- } \\
\text { arbeitet (Ressourcenaktivierung/-aktualisierung, d.h. expli- } \\
\text { zite inhaltliche Thematisierung oder prozessuale implizite } \\
\text { Aktivierung) und andererseits werden neue bzw. fehlende } \\
\text { Ressourcen und Lösungen gemeinsam erarbeitet. }\end{array}$ & $\begin{array}{l}\text { (17) [...] mit welchen, vielleicht auch Empfind- } \\
\text { samkeiten gehst du auf diesem Weg, ja, in den } \\
\text { nächsten Wochen, • • auch nochmal bewusster } \\
\text { um, auf dem Weg dorthin. }((1,5 \mathrm{~s})) \text { Auf dem } \\
\text { Weg dorthin, zu diesem }(1,5 \mathrm{~s})) / \text { wo du schon sehr } \\
\text { nah dran bist/ ((lacht)) Jaa. Ja! ‥ Er is auch jetz } \\
\text { ja grade da Hm̌ Ja. ((6s)) ((unv., 1s)) mit dem, } \\
\text { wos auch diese Empfindsamkeiten, wie willst } \\
\text { du mit denen umgehen? Auch die nächsten } \\
\text { Wochen. }((2,5 s)) \text { Welche Haltung, •• welchen • } \\
\text { • / mit welcher Einstellung, mit welchen Ge- } \\
\text { fühlen/ (KL_1-2) }\end{array}$ \\
\hline $\begin{array}{l}\text { (Konkrete) Lösungsstrategien entwickeln - in einem } \\
\text { letzten Schritt werden konkrete Lösungsstrategien oder } \\
\text { Maßnahmen kollaborativ entwickelt bzw. sollen sie für } \\
\text { Klient*innen realisierbar werden. Die Ressourcen werden } \\
\text { handhabbar gemacht, es soll (im Anschluss) ein Transfer, } \\
\text { d.h. eine Übertragung auf den gewünschten beruflichen } \\
\text { Kontext, stattfinden. }\end{array}$ & 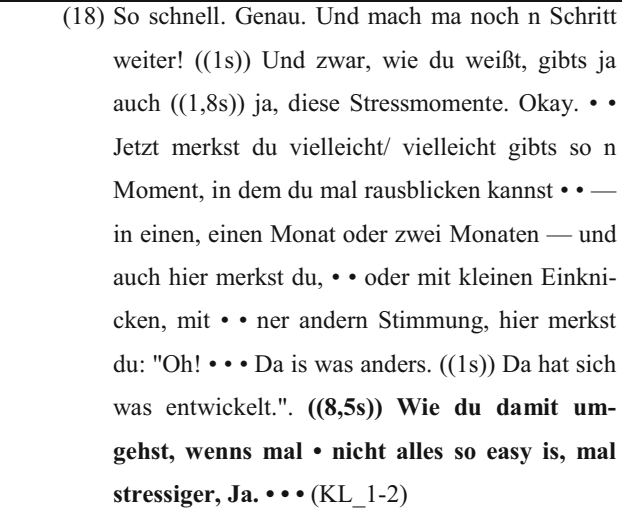 \\
\hline $\begin{array}{l}\text { (Konkrete) Lösungsstrategien anbieten - in diesem Fall } \\
\text { werden die Lösungsstrategien nicht kollaborativ (und auf- } \\
\text { bauend) entwickelt, sondern von Coaches angeboten bzw. } \\
\text { den Klient*innen an die Hand gegeben. Sie gehören zum } \\
\text { „Methodenkoffer“ der Coaches und werden manchmal } \\
\text { auch vorab (z.B. basierend auf einem Telefonat oder der } \\
\text { vorangehenden Sitzung) vorbereitet und als Lösungsme- } \\
\text { thode/-strategie den Klient*innen angeboten. Hier zeigt } \\
\text { sich das Spektrum von Coaching (angesiedelt im Span- } \\
\text { nungsfeld zwischen Therapie und Beratung) auf. }\end{array}$ & $\begin{array}{l}\text { (19) ((ea)) Genau. Und was hilft Ihnen da- • -bei, } \\
\text { das zu tun? Das ist ja genauso die Kunst, ne? • } \\
\text { Einerseits ist das Gefühl da • "Ich schaff s nicht. } \\
\text { Gott, die, } • \text { die Zeit ist so kurz, die Decke ist zu } \\
\text { kurz t-t-t-t-t-t.". ((1,3s)) Äh ja? Äh das hm äh, ne, } \\
\text { eher so: "Ich kann mir gar nicht das leisten, m- } \\
\text { hm.". So. • Also was hilft Ihnen dann? • • So zu } \\
\text { sagen: "Grade dann geh ich jetzt • rechtzeitig } \\
\text { nach Hause.". "Grad dann nehm ich mir jetzt Zeit } \\
\text { für Sport, oder für Ausgleich, oder...". • • Was } \\
\text { hilft Ihnen da, das dann zu tun? ((2,6s)) Oder } \\
\text { ist es so, dass Sie sagen: "Wenn ich s merke, } \\
\text { dann u ich..." , dann machen Sie das auch. Das } \\
\text { ist also nicht • die... (KL_3-4) }\end{array}$ \\
\hline
\end{tabular}


Abb. 7 Sequenzorganisationsmodell lösungsorientierter Fragen nach Kabatnik et al. (2019)

\begin{tabular}{|ll|}
\hline 0-Position & eingeschränkte Handlungsmächtigkeit des Patienten thematisch \\
1-Position & Lösungsorientierte Frage \\
2-Position & dispräferierte/suboptimale/korrekturbedürftige Antwort \\
3-Position & Insistenz des Therapeuten (oder Themenwechsel) \\
4-Position & Zustimmung/Ablehnung/(Ambivalenz)/optimierte Antwort \\
\hline
\end{tabular}

können so die mentale Verfassung ihrer Klient*innen einschätzen und wesentliche Informationen über sie gewinnen. Allerdings wird den Klient*innen von Beginn an eine Lösungsfindungskompetenz zugetraut. Diese kann auch von Beginn des Prozesses (ohne negative Auswirkungen auf Klient*innen) ausgetestet werden. Weiter können durch LoF Vorgehensweisen und Äußerungen von Klient*innen implizit durch die*den Coach kritisiert werden, wie z.B. in Mir ist nochmal wichtig, $((2,8 s))$ wir können mehr bewegen und Sie können mehr bewegen, wenn Sie schauen: Wo haben Sie noch Möglichkeiten? Ne? (KL3_2-3). Durch nochmal in Mir ist nochmal wichtig und noch in Wo haben Sie noch Möglichkeiten? wird markiert, dass bereits eine klient*innenseitige Antwort erfolgte, die durch die Coach jedoch (noch) nicht als adäquat eingestuft wurde, sodass eine weitere und veränderte Antwort konditionell relevant gesetzt wird und dies im Gespräch weiter vertieft werden kann.

Im Unterschied zu den therapeutischen Diagnosegesprächen findet sich in den Coaching-Gesprächen von Beginn an eine starke Fokussierung auf Lösungsfindung/-orientierung, was sich auch in den quantitativen Ergebnissen der Untersuchungen - mit 27 LoF häufiger am Ende der Therapie-Sitzung und $105 \mathrm{LoF}$ im Coaching ungefähr gleichmäßig über die drei Gesprächsphasen verteilt (vgl. oben) - spiegelt. Es geht im Coaching also nicht darum, generell wieder Handlungsfähigkeit herzustellen, sondern eine mangelnde Fähigkeit zur Handlungsentscheidung auszuloten und einen Handlungswiderstand zu überwinden. Es wird ein handlungsfähigeres Gegenüber als in der Psychotherapie angenommen, das bereits zu Beginn des Prozesses über Lösungsfindungskompetenz verfügt. So stehen die meisten Handlungen von Coaches im Dienste der Lösungsfindung/-orientierung und es erscheint notwendig, den Fragetyp LoF und die Funktion „Lösungsorientierung“ weiter zu differenzieren: Ausgehend von bisherigen Publikationen zu LoF und ersten empirischen Erkenntnissen im Zusammenhang mit einer umfassenden Fragentypologie im

\footnotetext{
${ }^{7}$ Im Kontext eines internationalen und interdisziplinären Forschungsprojekts zu Fragesequenzen im Coaching werden mehrere Qualifizierungsarbeiten, u. a. mit dem Fokus einer Fragentypologie sowie einer Fragensequenztypologie für den Interaktionstyp Coaching verfasst (https://questions-in-coaching.aau.at/).
}

Coaching (über den Typ Lösungsorientierte Frage hinaus) ${ }^{7}$ wird deshalb folgende funktionale Differenzierung ${ }^{8}$ von Lösungsfindung/-orientierung vorgeschlagen (Abb. 6).

Als übergeordnete Gemeinsamkeit setzen $\mathrm{LoF}$ in allen Fällen bzw. in all ihren Funktionen eine Ratifikation seitens der Klient*innen und Patient*innen konditionell relevant, wodurch existierende klient*innen- und patient*innenseitige Konzepte und Vorstellungen bzgl. der angestrebten Ergebnisse im Coaching und in der Psychotherapie identifiziert, geprüft und eingestuft werden können (s. Kabatnik et al. 2019; Graf und Kabatnik im Druck).

\subsection{Kontext von LoF im Vergleich}

In Bezug auf gesprächsstrukturelle Aspekte sind LoF in den therapeutischen Diagnosegesprächen meist präterminal zu verorten (vgl. Oelschläger 2017, S. 39): so wurden im Psychotherapie-Korpus 18 der 27 (rund 66,7\%) LoF im letzten Drittel des Gesprächs identifiziert. Nur in neun von 27 Fällen (rund 33,4\%) wurden LoF im ersten Drittel der Gespräche realisiert, was mit der Handlungsrationale der Psychotherapie zusammenhängen könnte. So muss im therapeutischen Kontext das Problem der Patient*innen im Gespräch zunächst erst ausführlich thematisiert und exploriert werden. Erst nach solch ausführlicher Problemexploration und -würdigung kann die Entwicklung von Lösungen angestrebt werden. Der Gebrauch von LoF ist somit stark von der interaktionalen und thematischen Entwicklung des Therapiegesprächs abhängig - sie werden deshalb im therapeutischen Kontext erst dann realisiert, nachdem die oftmals als emotional schwierig markierten Themen hinreichend besprochen wurden (vgl. Bröcher 2017, S. 34). In diesem Zusammenhang entwickelten Kabatnik et al. (2019) auf der Basis des oben beschriebenen Therapie-Korpus bereits ein sequenzielles Stufenmodell, das musterhaft die Organisation der Sequenzen im Kontext von Lösungsorientierten Fragen im therapeutischen OPD-Gespräch demonstriert (s.a. Läpple et al. 2021, S. 40; Abb. 7).

\footnotetext{
8 Wir bedanken uns vielmals bei Frédérick Dionne, Melanie Fleischhacker und Gundula Fofana für die Bereitstellung ihrer aktuellen Erkenntnisse bzgl. der funktionalen Ausdifferenzierung von LoF im Coaching.
} 
Tab. 1 Idealtypisches Beispiel einer lokalen Problembearbeitung in der Psychotherapie (vgl. Spranz-Fogasy et al. 2018, S. 126)

\begin{tabular}{|c|c|c|}
\hline $\begin{array}{l}\text { Sequenzposition } \\
\text { und Sprecher*in }\end{array}$ & Gesprächsausschnitt & Sequenztyp \\
\hline $0: \mathrm{P}$ & Anzeige von low agency durch P: Abhängigkeit von anderen & $\begin{array}{l}\text { Eingeschränkte } \\
\text { Handlungsfähigkeit } \\
\text { thematisch }\end{array}$ \\
\hline 1: $\mathrm{T}$ & $\begin{array}{l}((\mathrm{schmatzt}))^{\circ} \mathrm{h} \text { wie hätten sie_s denn gern }(1.22) \text { wenn sie }(.)^{\circ} \mathrm{h}(.) \text { sagen würden }(0.91) \text { (was/das) (.) } \\
\text { was sie an sich verändern können }(0.38) \text { ich mein jetz nich die beschwerden des is klar }\end{array}$ & $\begin{array}{l}\text { Lösungsorientierte } \\
\text { Frage }\end{array}$ \\
\hline 2: $\mathrm{P}$ & $\begin{array}{l}\text { was ich [an mir ver]ändern soll }(2.28) \mathrm{hh}^{\circ}(0.5) \text { ähm }(0.23)((\mathrm{schmatzt})) \text { ob ich des jemals verändern } \\
\left.\text { kann dass ich }(1.89) \mathrm{hm} \text { hm ungern alleine bin des weiß ich nich }{ }^{\circ} \mathrm{hh}\right] \text { ich weiß auch nich ob ich_s } \\
\text { (darauf) also also ob ich_s ändern will weil (.) äh ich genieß des ja (wenn) ich (1.11) leuten die mir } \\
\text { wichtig sin irgendwa (.) irgendwas zu unternehmen oder }{ }^{\circ} \mathrm{h}(.) \text { dass die bei mir sin oder }(1.77)\end{array}$ & $\begin{array}{l}\text { Dispräferierte Ant- } \\
\text { wort (non-answer } \\
\& \text { answer-like refo- } \\
\text { cusing response) }\end{array}$ \\
\hline 3: $\mathrm{T}$ & $\begin{array}{l}((\text { schnalzt })) \text { aber sie laufen ja schon gefahr }{ }^{\circ} \mathrm{h}(0.37) \text { ähm }(0.62) \text { sich immer wieder von andern abhän- } \\
\text { gig zu machen da[durch ] [dass ]jemand da sein muss wie so ne infusion }{ }^{\circ} \mathrm{h} \text { un_wenn die abgenom- } \\
\text { men wird dann ham sie angst dass }(.) \text { dass es schlimm wird }\end{array}$ & $\begin{array}{l}\text { Rephrasing formu- } \\
\text { lation }\end{array}$ \\
\hline 4: $\mathrm{P}$ & $\begin{array}{l}\left((.) \text { ja }(0.39)((\text { schmatzt }))^{\circ} \mathrm{hh} \text { ja des stimmt also ich erhoff mir schon dass ich irgendwo halt auch selbst- }\right. \\
\text { ständig sein kann dass ich }(0.55)((\text { schmatzt })) \text { un_eigenständig (.) un ohne ängste un ohne heimweh } \\
\text { äh [nenn ich_s jetz ]mal }{ }^{\circ} \mathrm{h}(.) \text { irgendwo hingehn kann meine (.) eigenen ziele verfol[gen ]oder so) }\end{array}$ & Optimierte Antwort \\
\hline
\end{tabular}

In Bezug auf die Handlungsfähigkeit der Patient*innen ermitteln Spranz-Fogasy et al. (2018) und Kabatnik et al. (2019), dass LoF an eine eingeschränkte Handlungsfähigkeit, also an eine niedrige Agency (vgl. Marciniak 2017) von Patient*innen anknüpfen (Position 0). Die Anknüpfungspunkte mit reduzierter Handlungsunfähigkeit befinden sich sowohl thematisch im behandelten Problemfeld als auch in den unmittelbar vorhergehenden Gesprächssequenzen oder können auch weiter zurückliegende Sequenzen aufgreifen. LoF knüpfen dabei inhaltlich entweder direkt an das Thema der Präsequenz(en) an $(n=23$, $85,2 \%)$ - wobei patient*innen- oder therapeut*innenseitige Äußerungen wiederaufgenommen werden können - oder die Therapeut*innen initiieren selbst ein neues und aus ihrer Sicht relevantes Thema $(n=4,14,8 \%)$ (Spranz-Fogasy et al. 2018, S. 123). So bringt etwa die Patientin in Gespräch T2-4 selbst das Thema wiederkehrender Ohnmachtszustände ein und kommt anschließend auf ihr Hauptproblem, nämlich den Mangel an Konsequenz: ich kann nicht wirklich konsequent sein (00:43:32). Dies wird vom Therapeuten in einer LoF lexikalisch wiederaufgenommen: wo würden sie sich wünschen, dass sie konsequenter wären (00:43:44 bis 00:43:49).

Das Sequenzorganisationsmodell nach Kabatnik et al. (2019) illustrieren wir für den vorliegenden Beitrag anhand des Beispiels T3-1 aus Spranz-Fogasy et al. (2018):

Der Patient zeigt in Sequenzposition 0 niedrige Agency ${ }^{9}$ an, weil er nur glücklich sein kann, wenn er seine Freude mit einer Person teilen kann. Der Therapeut erklärt dem Pa-

\footnotetext{
${ }^{9}$ Die niedrige Agency von Patient*innen in der Therapie bezieht sich auf persönlichkeitsumfassende Kontexte und auf weitaus schwierigere Problemfelder, wie z.B. wiederkehrende Suizidgedanken, Hemmungen, sich in der Therapie zu öffnen oder maladaptive Abhängigkeitsverhältnisse in Partnerschaften (vgl. Kabatnik et al. 2019, S. 156; Marciniak 2017, S. 41).
}

tienten daraufhin die Gefahren einer Abhängigkeit von Anderen. In Sequenzposition 1 wird die LoF gestellt, auf die dann in Sequenzposition 2 eine response der Patient*innen folgt. Im vorliegenden Psychotherapie-Korpus werden hierbei in allen Fällen dispräferierte korrekturbedürftige Antworten gegeben. In Sequenzposition 3 schließen Therapeut*innen - meist in Form einer formulation - an und insistieren oder refokussieren so auf ein anderes Thema. In Abhängigkeit davon, wie Therapeut*innen ihre Reaktion formulieren, können die Patient*innen in Sequenzposition 4 zustimmend, ablehnend oder ambivalent zur therapeutischen Präsequenz Stellung beziehen oder eine optimierte Antwort, z. B. auf die gestellte LoF, produzieren ${ }^{10}$ (Tab. 1).

Dieses Beispiel aus Spranz-Fogasy et al. (2018, S. 126) stellt einen idealtypischen Fall der lokalen Problembearbeitung mittels LoF in der Psychotherapie dar, die durch Rekursion einen Mechanismus der Wissensumstrukturierung und Bearbeitung von Widerstand darstellen kann (vgl. Kabatnik et al. 2019).

Betrachtet man den Gesprächsverlauf bzw. den Kontext von LoF, so ergeben die Analysen der Coaching-Daten, dass sie im ersten $(n=36$; rund $34 \%)$, zweiten $(n=37$; rund $35 \%)$ und dritten Drittel $(n=32$; rund $30 \%)$ der Gespräche auftreten. Aufgrund der Tatsache, dass die Beteiligten beim Stellen von Lösungsorientierten Fragen hinsichtlich der Aushandlung eines Problems oder eines Anliegens an einem - wenn auch nur vorläufigen oder für die aktuelle Sitzung geltenden - Endpunkt angelangt sind (vgl. SpranzFogasy 2020, S. 64), erscheint das Stellen der LoF, d.h.

\footnotetext{
10 Das Sequenzmodell beschreibt einen idealtypischen Bearbeitungsverlauf Lösungsorientierter Fragen im Psychotherapie-Gespräch; d.h. die einzelnen Sequenzpositionen sind nicht immer in dieser Organisation vorzufinden, sondern können auch in schleifenartigen Verläufen realisiert werden (vgl. Oelschläger 2017, S. 50).
} 
eine Lösungsentwicklung, am Ende der Sitzung und somit nach dem Diagnostizieren bzw. Klären und Bearbeiten des Problems aus handlungslogischer Perspektive erwartbar. Auffallend ist dagegen, dass im Coaching LoF gleichermaßen zu Beginn und im mittleren Teil eines Coaching-Gesprächs vorkommen. Werden Fragen am Anfang der Sitzung gestellt, können sie zur inhaltlichen Planung der Coaching-Sitzung beitragen, wie z.B. durch wo $\bullet$ zieht es Sie jetzt so am meisten hin. Oder was wäre als nächstes für Sie so ein guter/ $\bullet$ guter Schritt so für heute (KL2_12). Auf diese Weise zielen LoF auf Themen, die für die jeweilige Coaching-Sitzung von Bedeutung sind, d.h. sie elizitieren thematische Relevanzmarkierungen und weisen dabei gesprächsstrukturierende Funktionen auf (vgl. Graf und Kabatnik im Druck). Aber auch am Ende der Sitzung können ähnliche Fragen auftreten, zum Beispiel zur Kontrolle, ob ein Gegenstand der Sitzung ausreichend behandelt wurde und/oder um herauszufinden, welche Themen noch behandelt werden sollen, wie z. B. in $((1,5 s))$ Blick auf die Uhr. Wir haben also jetzt noch $\bullet$ ne halbe Stunde, ja? ••Was wär denn, sagen wir, bezogen auch auf diie, $\bullet \bullet$ die (Ziele) was wär heute noch wichtig zu besprechen? $((2,3 s))$ Erstmal ist so die Frage: Haben Sie das Gefühl, das sind die zwei wichtigsten Elemente, die wir bis jetzt besprochen haben? (KL2_1-3). Die Ursache dieser Zielgerichtetheit liegt einerseits im spezifischen Gesprächsformat Coaching selbst, da dieses auf möglichst hohe Effizienz und das schnelle Generieren von (messbaren) Lösungen ausgerichtet ist (siehe oben). Andererseits liegt dem Coaching der Gedanke zugrunde, dass Klient*innen einen bestimmten Grad an Handlungsfähigkeit aufweisen; so haben diese oftmals bereits konkrete Ideen und Vorstellungen davon, wie in der jeweiligen Sitzung ein Thema behandelt werden sollte. Gerade die Funktion von Lösungsorientierten Fragen Klient*innen in die thematische Gestaltung einzubinden, mag auch dem speziellen Klientel „Führungskräfte“ und ihrem Anspruch und Selbstverständnis geschuldet sein. Allgemein weist die hier dokumentierte Praktik des durchgängigen Stellens Lösungsorientierter Fragen die durchaus kritisch zu hinterfragende „einseitige Lösungsorientierung im Coaching“" (Künzli, persönliches Gespräch) nach, also die auf Effizienz ausgerichtete und sehr schnelle Fokussierung auf Lösungen bei reduzierter Bearbeitung von Problemursachen.

In Bezug auf ihre kontextuelle Anknüpfung zeigt sich, dass LoF inhaltlich entweder direkt am vorhergehenden Thema anschließen - wobei Äußerungen der Coaches $(n=24$, rund $23 \%)$ oder der Klient*innen $(n=37$, rund $35 \%$ ) wiederaufgenommen werden können - oder dass die Coaches selbst ein neues Thema einführen $(n=36$, rund $34 \%$ ). Den Fragen geht zudem stets eine Problemthematisierung voraus, die von niedriger Handlungsfähigkeit der Klient*innen gekennzeichnet ist (vgl. Graf und Kabatnik im Druck; zu Psychotherapie Marciniak 2017) und zu deren Lösung eben der Einsatz von LoF dienen soll. Allerdings bezieht sich die im Coaching behandelte niedrige Agency zumeist auf sehr konkrete (berufliche) Kontexte, wie den Wunsch nach mehr Urlaub (KL1 3) oder einer genaueren Bearbeitung von Aufgaben durch Angestellte (KL2_1-1) sowie den Umgang mit Konflikten im privaten Bereich (wobei Lösungen hierbei im weiteren Gesprächsverlauf dann auf berufliche Konflikte übertragen werden) (KL3_12).

Ein Sequenzorganisationsmodell für LoF im Coaching in Analogie zu dem hier für die Psychotherapie-Daten vorgestellten Modell ist in Vorbereitung (Kabatnik und Graf in Vorbereitung).

\section{Das Interaktionstyp-spezifische Veränderungspotential von LoF in Psychotherapie und Coaching - Schlussfolgerungen und Ausblick}

Coaching und Psychotherapie werden als helfende Formate initiiert, um in und durch eine Reihe von Gesprächen zwischen Coach/Therapeut*in und Klient*in/Patient*in eine Veränderung bzw. eine Lösung für die Probleme oder Anliegen zu generieren, wegen derer die helfenden Interaktionen von den Beteiligten ursprünglich initiiert wurden. Während das zugrundeliegende Ziel einer Veränderung den beiden Formaten gemeinsam ist, ist der Weg dahin (aber auch der Typ und die Dimension von Veränderung) in vielerlei Hinsicht unterschiedlich. So argumentiert etwa Fischer-Epe (2000, S. 186) in diesem Zusammenhang für Coaching, ,(u)nser Ziel ist es nicht, Ursachen für Probleme zu finden, sondern Ziele und Lösungen in der Zukunft zu erreichen". Die sofortige Fokussierung auf (schnelle) konkrete Lösungen für mehr oder weniger klar umrissene (berufliche) Herausforderungen ohne eine detaillierte Exploration der Ursachen im Unterschied zu einer umfassenden und tiefgehenden Erforschung der in der Vergangenheit liegenden Ursprünge und Ursachen der Patient*innen-Probleme stellt den zentralen Unterschied zwischen beiden Formaten dar. Dieser manifestiert sich in unterschiedlich gestalteten sprachlich-interaktiven Wegen, geprägt von den professionellen Agenden der Coaches bzw. der Therapeut*innen und der dem jeweiligen Format zugrundeliegenden Handlungsrationale bzw. Veränderungstheorie. Dieser Weg manifestiert sich auf der Ebene der Interaktion lokal und in-situ in der sequentiellen turn-by-turn Ko-Konstruktion entlang des konkreten Gesprächs und wird durch die Interventionen von Coach bzw. Therapeut*in maßgeblich geprägt (siehe oben). LoF sind dabei in beiden Formaten ein zentrales Instrument bzw. eine zentrale diskursive Praktik; sie haben das Ziel, Lösungen für Probleme und Konflikte von Klient*innen 
und Patient*innen zu elizitieren. Dieses Ziel wird von den Interaktant*innen im geschützten Rahmen des professionellen Hilfe-Gesprächs ko-konstruiert - die sprachlich durch den hypothetischen, und dadurch entlastenden, Modus markierten Fragen entwerfen prospektiv Lösungsmöglichkeiten und können so Veränderungspotenziale generieren.

Während der Fragetyp LoF durch seine formal-sprachliche, lexikalische und modale Gestaltung bereits ein inhärentes sprachlich-interaktives Lösungspotential aufweist, das als Interaktionstyp-unspezifisch betrachtet werden kann (vgl. Graf und Kabatnik im Druck), galt der vorliegende Beitrag der Frage, welches Interaktionstyp-spezifische, lokale Veränderungspotential Lösungsorientierte Fragen im Coaching - im Unterschied zur Psychotherapie - aufweisen. Zusammenfassend kann dieses so formuliert werden:

- In den Coachingdaten finden sich fast vier Mal so viele LoF wie in den OPD-Daten; da jede LoF die Klient*innen zur Entwicklung von Lösungen einlädt, indem sie eine entsprechende response konditionell relevant setzt, kann die aufgezeigte Häufigkeit als Ausdruck des lokalen, Interaktionstyp-spezifischen Veränderungspotentials interpretiert werden.

- Die Zentralität der Lösungsorientierung und -konstruktion im Coaching wird dabei durch folgende vorläufige Differenzierung des Fragetyps LoF bzw. seiner Funktionen (und in weiterer Folge auch eine Differenzierung der Fragetypen wie etwa Fragen zum Agenda-setting) (vgl. Dionne \& Graf in Vorbereitung) abgebildet: zunächst gilt es, (vorhandene) Lösungsressourcen aufzudecken, Lösungswünsche zu explorieren und/oder (fehlende) Lösungsressourcen zu be-/erarbeiten, um darauf aufbauend, (konkrete) Lösungsstrategien mit den Klient*innen zu entwickeln. Alternativ bieten Coaches auch (konkrete) Lösungsstrategien an, wenn von Klient*innen-Seite selbst keine Vorschläge oder Ideen eingebracht werden.

- Lösungsorientierte Fragen treten im Coaching entlang der gesamten Sitzung in einer relativ ausgewogenen Verteilung auf, d.h. Lösungen gedanklich möglich zu machen, steht entlang des gesamten Gesprächs im Fokus. Lösungen beinalten hierbei auch vermehrt die thematische und interaktive Gestaltung des Coachings und realisieren somit die endemischen Aufgaben helfender Gespräche, „Beziehungsgestaltung“ und „Wissensgenese und -vermittlung“" (siehe Kap. 2).

- Lösungsorientierte Fragen stellen eine zentrale diskursive Praktik im Kontext der sprachlich-diskursiven Realisierung der working alliance im Coaching dar; gerade durch das aktive Einbinden der Klient*innen durch LoF bei der Agenda-Gestaltung wird (in Zügen) eine nichthierarchische Beziehungsgestaltung als auch die Attribution einer grundsätzlichen Handlungsfähigkeit auf Klient*innen-Seite zum Ausdruck gebracht. Insbesondere die Vielzahl an LoF, die gestellt werden, zeigt dies auf, da sie ja Verantwortung an die Klient*innen abgeben, ihnen dabei Lösungsfindungskompetenz zusprechen und LoF somit die primäre Funktion im Coaching, nämlich eine tatsächliche Lösungsfindung zu generieren - im Unterschied zur Psychotherapie, wo die primäre Funktion von LoF die Überprüfung von Bewusstseinszuständen und Perspektiven der Patient*innen sowie ihrer Fähigkeit zur Lösungsfindung (siehe oben) ist - auch auf der Beziehungsebene ausdrücken. Da die working alliance einen der zentralen Wirkfaktoren im Coaching darstellt (Graf und Jautz im Druck), d.h. seiner globalen Effizienz und Wirksamkeit zugrunde liegt, können LoF in ihrem lokalen, in-situ Beitrag zur working alliance somit auch als Bindeglied zwischen lokaler und globaler Wirksamkeit interpretiert werden.

Der Fokus auf die globale Wirksamkeit von Lösungsorientierten Fragen im Coaching, allerdings im Kontext einer supra-session courses of action (Bercelli et al. 2013), d.h. also ein Fokus auf die interaktive Entwicklung der im Sequenzorganisationsmuster (siehe Tab. 1) dargelegten einzelnen Sequenz entlang der gesamten Coaching-Sitzungen bzw. gesamter Coaching-Prozesse und der Einfluss dieser Entwicklung auf die Wirksamkeit von Coaching stellt einen nächsten logischen Forschungsschritt dar (vgl. Kabatnik und Graf in Vorbereitung). Darüber hinaus gilt es auch, die Differenzierung der Funktionen und Fragetypen zur Lösungsfindung und -orientierung im Coaching und ihre interaktive, sequenzielle und kontextuelle Gestaltung entlang gesamter Coaching-Sitzungen und kompletter Coaching-Prozesse differenziert zu dokumentieren und zu analysieren. Und schließlich sollen die Erkenntnisse der gesprächsanalytischen Erforschung von Lösungsorientierten Fragen im Coaching als zentrale diskursive Praktik des dezidiert auf das schnelle Erarbeiten von konkreten Lösungen für die (berufliche) Zukunft der Klient*innen ausgerichteten Beratungsformats für die Coaching-Praxis im Kontext von Handlungsempfehlungen aufbereitet werden (vgl. Graf und Kabatnik in Vorbereitung; Tab. 2). 
Tab. 2 Verwendete cGAT-Transkriptionskonventionen (Minimaltranskript)

\begin{tabular}{|c|c|}
\hline Wörter & $\begin{array}{l}\text { Literarische Umschrift } \\
\text { (keine Großbuchstaben, kein Apostroph, kein } \\
\text { Bindestrich, keine Diakritika) }\end{array}$ \\
\hline Simultanpassagen & [ ] \\
\hline Verzögerungssignale & äh \\
\hline Rezeptionssignale & $\begin{array}{l}\mathrm{hm} \text { (einsilbig positiv) } \\
\mathrm{hmhm} \text { (zweisilbig positiv) } \\
\text { mh (einsilbig negativ) } \\
\text { mhmh (zweisilbig negativ) }\end{array}$ \\
\hline $\begin{array}{l}\text { Vor- und Nach- } \\
\text { laufelemente }\end{array}$ & ja, ne \\
\hline $\begin{array}{l}\text { Verschleifungen } \\
\text { zwischen Wör- } \\
\text { tern }\end{array}$ & $\begin{array}{l}\text { hab_s } \\
\text { gibt_s }\end{array}$ \\
\hline Unverständliches & $\begin{array}{l}\text { +++ (einsilbiges unverständliches Wort) } \\
++++++ \text { (zweisilbiges unverständliches Wort) } \\
\text { ((unverständlich)) (unverständliche Passage, } \\
\text { wenn länger als eine Sekunde mit Zeitangabe) }\end{array}$ \\
\hline $\begin{array}{l}\text { Vermuteter Wort- } \\
\text { laut }\end{array}$ & (glaube ich) \\
\hline Alternativlautungen & (ja/so) \\
\hline $\begin{array}{l}\text { Mikropausen } \\
\text { (unter } 0,2 \text { Sek. } \\
\text { Dauer) }\end{array}$ & (.) \\
\hline $\begin{array}{l}\text { Gemessene Pau- } \\
\text { sen }\end{array}$ & $(0.35)$ \\
\hline Einatmen & 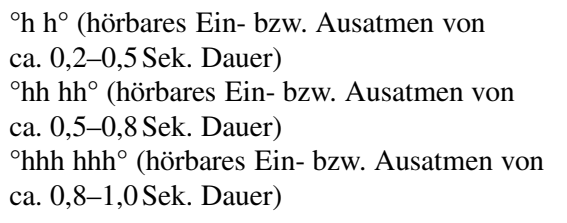 \\
\hline $\begin{array}{l}\text { Nonverbale } \\
\text { Handlungen } \\
\text { und Ereignisse } \\
\end{array}$ & $\begin{array}{l}((\text { lacht })) \\
((\text { räuspert sich }))(\text { wenn länger als eine Sekunde } \\
\text { mit Zeitangabe })\end{array}$ \\
\hline
\end{tabular}

Nach: Schmidt, Thomas/Schütte, Wilfried/Winterscheid, Jenny (2015). Abrufbar unter: https://ids-pub.bsz-bw.de/frontdoor/index/ index/docId/4616

cGAT Konventionen für das computergestützte Transkribieren in Anlehnung an das Gesprächsanalytische Transkriptionssystem 2 (GAT2)

Danksagung Wir bedanken uns bei Melanie Fleischhacker für die detaillierte Kommentierung einer früheren Version dieses Beitrags, bei Fabienne Wautsche für das professionelle proof reading und den beiden Gutachter*innen, die durch ihre wertvollen Anmerkungen die Qualität unseres Beitrags noch weiter verbessert haben.

Open Access Dieser Artikel wird unter der Creative Commons Namensnennung 4.0 International Lizenz veröffentlicht, welche die Nutzung, Vervielfältigung, Bearbeitung, Verbreitung und Wiedergabe in jeglichem Medium und Format erlaubt, sofern Sie den/die ursprünglichen Autor(en) und die Quelle ordnungsgemäß nennen, einen Link zur Creative Commons Lizenz beifügen und angeben, ob Änderungen vorgenommen wurden.

Die in diesem Artikel enthaltenen Bilder und sonstiges Drittmaterial unterliegen ebenfalls der genannten Creative Commons Lizenz, sofern sich aus der Abbildungslegende nichts anderes ergibt. Sofern das betreffende Material nicht unter der genannten Creative Commons Lizenz steht und die betreffende Handlung nicht nach gesetzlichen Vorschriften erlaubt ist, ist für die oben aufgeführten Weiterverwendungen des Materials die Einwilligung des jeweiligen Rechteinhabers einzuholen.

Weitere Details zur Lizenz entnehmen Sie bitte der Lizenzinformation auf http://creativecommons.org/licenses/by/4.0/deed.de.

\section{Literatur}

Arbeitskreis OPD (2014). Operationalisierte Psychodynamische Diagnostik OPD-2: Das Manual für Diagnostik und Therapieplanung. 3., überarb. Aufl. Bern: Hans Huber.

Bercelli, F., Rossano, F., \& Viaro, M. (2013). Supra-session courses of action in psychotherapy. Journal of Pragmatics, 57, 118-137.

Blumer, H. (1954). What is wrong with social theory? American Sociological Review, 19(1), 3-10. https://doi.org/10.2307/2088165.

Brinker, K., \& Sager, S. F. (2010). Linguistische Gesprächsanalyse: Eine Einführung (5. Aufl.). Erich Schmidt.

Bröcher, L. (2017). Lösungsorientierte Fragen in psychodiagnostischen Gesprächen. Die Funktionen der Testung, impliziter Kritik und Ermittlung tatsächlicher Lösungsmöglichkeit. Masterarbeit. Mannheim: Universität Mannheim.

Cavanagh, M., \& Buckley, A. (2014). Coaching and Mental Health. The Complete Handbook of Coaching, 2, 405-417.

Crowe, T. (2017). Coaching and psychotherapy. In T. Bachkirova, G. Spence \& D. Drake (Hrsg.), The SAGE handbook of coaching (S. 85-101). SAGE.

Deplazes, S. (2016). KaSyCo: Kategoriensysteme zur Analyse von Coachingprozessen. Kassel University Press.

Deppermann, A. (2008). Gespräche analysieren. Eine Einführung (4. Aufl.). VS

Deppermann, A. (2015a). Wissen im Gespräch: Voraussetzung und Produkt, Gegenstand und Ressource. InLiSt - Interaction and Linguistic Structures, 51, 1-29.

Deppermann, A. (2015b). Agency in Erzählungen über Gewalterfahrungen in Kindheit und Jugend. In C. Scheidt, G. Lucius-Hoene, A. Stukenbrock \& E. Waller (Hrsg.), Narrative Bewältigung von Trauma und Verlust (S. 64-75). Schattauer.

Deppermann, A. (2018). Was ist Gesprächs- bzw. Konversationsanalyse? Methodologische Grundlagen. GAIS - Gesprächsanalytisches Informationssystem. http://prowiki.ids-mannheim.de/bin/view/ GAIS/DatenErhebung. Zugegriffen: 25.11.2021.

Dionne, F., \& Graf, E. (in prep.). Agenda-Thematizing questions in executive coaching-A linguistic analysis. Journal of Pragmatics.

Drath, K. (2012). Coaching und seine Wurzeln. Erfolgreiche Interventionen und ihre Ursprünge. Haufe Gruppe.

Drew, P., \& Heritage, J. (1992). Analyzing talk at work: an introduction. In P. Drew \& J. Heritage (Hrsg.), Talk at work. Interaction in institutional settings (S. 3-65). Cambridge University Press.

Fischer-Epe, M. (2000). Coaching: Miteinander Ziele erreichen. Rowohlt.

Graf, E. (2019). The pragmatics of executive coaching. John Benjamins.

Graf, E. (2021). Helfen im Führungskräfte-Coaching als kommunikativer Aushandlungsprozess in verschiedenen Spannungsfeldern. In D. Böhringer, S. Hitzler \& M. Richter (Hrsg.), „Helfen “ als situatives und organisationales Phänomen (S. 131-157). transkript.

Graf, E., \& Jautz, S. (accepted for publication). Linguistic insights into coaching, working alliance and client design as discursive achievements during first sessions. In C. Scarvaglieri, E. Graf \& T. Spranz-Fogasy (Hrsg.), The pragmatics of relationship building in helping professions. John Benjamins.

Graf, E., \& Kabatnik, S. (in prep.). Fragehandlungen im Coaching eine evidenz-basierte Handreichung für die Praxis.

Graf, E., \& Kabatnik, S. (accepted for publication). W]ie und was können Sie noch besser $\bullet$ organisieren, um • • auch Freiräume sich zu sichern? [...] Wo kann das irgendwie noch effektiver, 
$((1,2 \mathrm{~s})) \mathrm{ja}, \bullet \bullet$ gestaltet werden? - Lösungsorientierte Fragen im Führungskräfte-Coaching aus gesprächsanalytischer Perspektive. Zeitschrift für Angewandte Linguistik. Under review.

Graf, E., \& Pawelczyk, J. (2014). The interactional accomplishment of feelings-talk in relationship-focused integrative psychotherapy and executive coaching: Same format, different function? In E. Graf, M. Sator \& T. Spranz-Fogasy (Hrsg.): Discourses of Helping Professions (S. 59-89).

Graf, E., \& Spranz-Fogasy, T. (2018a). Welche Frage, wann und warum? Eine qualitativ-linguistische Programmatik zur Erforschung von Frage-Sequenzen als zentrale Veränderungspraktik im Coaching. Coaching | Theorie Praxis. https://doi.org/10.1365/ s40896-018-0021-4.

Graf, E., \& Spranz-Fogasy, T. (2018b). Helfende Berufe - Helfende Interaktionen. In K. Birkner \& N. Janich (Hrsg.), Handbuch Text und Gespräch (S. 418-442). De Gruyter.

Graf, E. \& Wastian, M. (2014). Coaching macht (Hoch)Schule, wenn ...: Erkenntnisse und Empfehlungen zur Qualitätssicherung im Coaching. Themenheft „Qualitätssicherung bei Präventions- und Interventionskonzepten in der Arbeits- und Organisationspsychologie", Wirtschaftspsychologie.

Graf, E., Dionne, F., \& Spranz-Fogasy, T. (2020). How to unravel the local and global change potential of questioning sequences in executive coaching?-A call for interdisciplinary research. Scandinavian Studies in Language, 11(1), 214-238. Special Issue "Questioning Questions in Language, Culture and Cognition" (edited by S. Borchmann, S. Mortensen \& L. Tranekaer).

Graf, E., Sator, M., \& Spranz-Fogasy, T. (Hrsg.). (2014). Discourses of helping professions. John Benjamins.

Graf, E., Scarvaglieri, C., \& Spranz-Fogasy, T. (Hrsg.) (2019). Pragmatik der Veränderung. Problem- und lösungsorientierte Kommunikation in helfenden Berufen. Studien zur Pragmatik. Tübingen: Narr.

Günthner, S. (2019). Kultur-in-kommunikativen-Praktiken', Kommunikative Praktiken zur Übermittlung schlechter Nachrichten in onkologischen Aufklärungsgesprächen. In J. Schröter, S. Tienken, Y. Ilg, J. Scharloth \& N. Bubenhofer (Hrsg.), Linguistische Kulturanalyse (S. 269-292). De Gruyter.

Günthner, S. Relationship building in oncological doctor-patient interaction (accepted for publication). The use of address forms as 'tie signs. In C. Scarvaglieri, E. Graf \& T. Spranz-Fogasy (Hrsg.), The pragmatics of relationship building in helping professions. John Benjamins.

Jautz, S. (2017). Immer auf Augenhöhe? Ein Blick in den sprachlichen Werkzeugkoffer im Coaching. In M. Dräger \& M. Kuhnhenn (Hrsg.), Linguistisches Wissen in Weiterbildungen zur Kommunikationskompetenz (S. 47-64). Peter Lang.

Kabatnik, S., Nikendei, Ch , Ehrenthal, J.C., \& Spranz-Fogasy, T. (2019). The Power of LoF. Veränderung durch Lösungsorientierte Fragen im psychotherapeutischen Gespräch. In E. Graf, C. Scarvaglieri \& T. Spranz-Fogasy (Hrsg.), Pragmatik der Veränderung. Problem- und lösungsorientierte Kommunikation in helfenden Berufen (S. 147-175). Gunter Narr.

Kabatnik, S., \& Graf, E. (in prep.). Veränderung durch Lösungsorientierte Fragen - eine longitudinale Untersuchung von CoachingProzessen.

Köller, W. (2004). Perspektivität und Sprache. Zur Struktur von Objektivierungsformen in Bildern, im Denken und in der Sprache. De Gruyter.

Kook, J. (2015). Agency in Arzt-Patient-Gesprächen: Zur interaktionistischen Konzeptualisierung von Agency. Peter Lang.

Kramer, U., \& Stiles, W. B. (2015). The responsiveness problem in psychotherapy: a review of proposed solutions. Clinical Psychology: Science and Practice, 22(3), 277-295.

Läpple, S., Nikendei, Ch , Ehrenthal, J.C., Kabatnik, S., \& SpranzFogasy, T. (2021). Therapeutische Reaktionen auf Patientenwi- derstand in psycho-diagnostischen Gesprächen - am Beispiel Lösungsorientierter Fragen. Verlag für Gesprächsforschung.

Lucius-Hoehne, G. (2012). Und dann haben wir's operiert'. Ebenen der Textanalyse narrativer Agency-Konstruktionen. In S. Bethmann, C. Helfferich, H. Hoffmann \& D. Niermann (Hrsg.), Agency. Qualitative Rekonstruktionen und gesellschaftstheoretische Bezüge von Handlungsmächtigkeit. Weinheim.

Mack, C., Nikendei, C., Ehrenthal, J.C., \& Spranz-Fogasy, T. (2016). [...] hab ich glaub ich die richtigen fragen gestellt." Therapeutische Fragehandlungen in psychodiagnostischen Gesprächen. OPAL. https://doi.org/10.14618/opal_03-2016

MacMartin, C. (2008). Resisting optimistic questions in narrative and solution-focused therapies. In A. Peräkylä, C. Antaki, S. Vehviläinen \& I. Leudar (Hrsg.), Conversation analysis and psychotherapy (S. 80-99). Cambridge University Press.

Marciniak, A. (2017). Agency in Lösungsorientierten Fragen im Psycho-diagnostischen Gespräch. Masterarbeit. Universität Mannheim.

Marciniak, A., Nikendei, C., Ehrenthal, J.C., \& Spranz-Fogasy, T. (2016). ...durch Worte heilen"-Linguistik und Psychotherapie. Sprachreport, 32(3), 1-11.

Middendorf, J., \& Salomon, L. (2017). 15. Coaching-Umfrage: Die Honorare steigen wieder. Wirtschaft \& Weiterbildung, 4(17), $38-41$.

Miller, K., \& Considine, J. (2009). Communication in the helping professions. In L. Frey \& K. Cissna (Hrsg.), The Routledge handbook of applied communication research (S. 405-428). Routledge.

Muntigl, P., \& Zabala, L.H. (2008). Expandable responses: how clients get prompted to say more during psychotherapy. Research on Language \& Social Interaction, 41(2), 187-226.

Oelschläger, E. (2017). Lösungsorientierte Fragen in psychodiagnostischen Gesprächen. Testen, Übertragen der Verantwortung und implizite Kritik. Masterarbeit. Universität Mannheim.

Pawelczyk, J., \& Graf, E. (2011). Living in therapeutic culture: feminine discourse as an agent of change. In L. Holmgreen \& I. Lassen (Hrsg.), Living with patriarchy-discursive constructions of gendered subjects across public spheres (S. 273-302). John Benjamins.

Peltier, B. (2010). The psychology of executive coaching. Theory and application. Routledge.

Peräkylä, A. (2013). Conversation analysis in psychotherapy. In J. Sidnell \& T. Stivers (Hrsg.), The handbook of conversation analysis (S. 551-574). Wiley-Blackwell.

Peräkylä, A. (2019). Conversation analysis and psychotherapy: identifying transformative sequences. Research on Language and Social Interaction, 52(3), 257-280.

Peräkylä, A., Antaki, C., Vehviläinen, S., \& Leudar, I. (Hrsg.). (2008). Conversation analysis and psychotherapy. Cambridge University Press.

Pick, I. (Hrsg.). (2017). Beraten ist Sprechen. Eine linguistische Typologie zu Beratungsgesprächen in verschiedenen Handlungsfeldern. Peter Lang.

Pick, I., \& Scarvaglieri, C. (2019). Helfendes Handeln. Zum Begriff sprachlichen Helfens und seinen Implikationen für Veränderung. In E. Graf, C. Scarvaglieri \& T. Spranz-Fogasy (Hrsg.): Pragmatik der Veränderung. Problem- und lösungsorientierte Kommunikation in helfenden Berufen. Tübingen: Narr. (S. 25-64).

Pick, I., \& Scarvaglieri, S. (2021). Helfen im Gespräch: Empirischer Vergleich der Hilfe in Rechtsberatung und Psychotherapie. In D. Böhringer, S. Hitzler \& M. Richter (Hrsg.), „Helfen“ als situatives und organisationales Phänomen. Transkript. in Druck.

Plank, F. (1986). Über den Personenwechsel und den anderer deiktischer Kategorien in der wiedergegebenen Rede. Zeitschrift für germanistische Linguistik, 14(3), 284-308.

Schegloff, E. (2007). Sequence organization in interaction. A primer in conversation analysis. Cambridge University Press. 
Schulz, F. (2015). Ein organisationstheoretischer Ansatz zur Erforschung diskursiver Prozesse im Management Coaching. Coaching | Theorie Praxis, 2, 1-7.

Seiger, C., \& Künzli, H. (2012). Schweizerischer Coaching-Markt 2011 aus Sicht von Coaches. Zürich: Zürcher Hochschule für Angewandte Wissenschaften.

Spranz-Fogasy, T. (2010). Vestehensdokumentation in der medizinischen Kommunikation: Fragen und Antworten im Arzt-PatientGespräch. In A. Deppermann, U. Reitemeier, R. Schmitt \& T. Spranz-Fogasy (Hrsg.), Verstehen in professionellen Handlungsfeldern (S. 27-116). Gunter Narr.

Spranz-Fogasy, T. (2020). Fragen und ihre Funktionen in psychotherapeutischen Gesprächen. In H. Gruber, J. Spitzmüller \& R. de Cillia (Hrsg.), Institutionelle und organisationale Kommunikation. Theorie, Methodologie, Empirie und Kritik (S. 39-69). Vandenhoeck \& Ruprecht.

Spranz-Fogasy, T., Ehrenthal, J.C., Graf, E., \& Nikendei, Ch (2019a). Die Bedeutung von Beispielnachfragen im Kontext von Veränderung: Elizitierungs- und Prozessierungstrategien im Vergleich von Therapie und Coaching. In E. Graf, C. Scarvaglieri \& T. Spranz-Fogasy (Hrsg.), Pragmatik der Veränderung. Problem- und lösungsorientierte Kommunikation in helfenden Berufen (S. 177-207). Gunter Narr.

Spranz-Fogasy, T., Graf, E., Ehrenthal, J. C., \& Nikendei, Ch (2019b). Beispiel-Nachfragen im Kontext von Veränderung: Elitizierungsund Prozessierungsstrategien in Psychotherapie und CoachingGesprächen. In E. Graf, C. Scarvaglieri \& T. Spranz-Fogasy (Hrsg.), Pragmatik der Veränderung. Problem- und lösungsorientierte Kommunikation in helfenden Berufen (S. 177-209). Gunter Narr.

Spranz-Fogasy, T., Kabatnik, S., \& Nikendei, Ch (2018). Wissenskonstitution durch Lösungsorientierte Fragen in psychodiagnostischen Gesprächen. In E. Hess-Lüttich (Hrsg.), Rhetorik und Medizin (S. 110-131). Mouton De Gruyter.
Steensig, J., \& Drew, P. (2008). Introduction: questioning and affiliation/disaffiliation in interaction. Discourse Studies, 10(1), 515.

Stiles, W. B. (2013). The variables problem and progress in psychotherapy research. Psychotherapy, 50(1), 33-41.

Tiittula, L. (2001). Formen der Gesprächssteuerung. In K. Brinker, G. Antos, W. Heinemann \& S.F. Sager (Hrsg.), Text- und Gesprächslinguistik: Ein internationales Handbuch zeitgenössischer Forschung (S. 1361-1374). Mouton De Gruyter.

Tracy, K., \& Robles, J. (2009). Questions, questioning, and institutional practices: an introduction. Discourse Studies, 11(2), 131-152.

Voutilainen, L., \& Peräkylä, A. (2016). Interactional practices of psychotherapy. In M. O'Reily \& J. Lester (Hrsg.), The Palgrave handbook of adult mental health (S. 540-557). Palgrave Macmillan.

Voutilainen, L., Peräkylä, A., \& Ruusuvuori, J. (2011). Therapeutic change in interaction: conversation analysis of a transforming sequence. Psychotherapy Research, 21(3), 348-365.

Voutilainen, L., Rossano, F., \& Peräkylä, A. (2018). Conversation analysis and psychotherapeutic change. In S. Pekarek Doehler, J. Wagner \& E. González-Martínez (Hrsg.), Documenting change across time: longitudinal studies on the organization of social interaction (S. 225-254). Palgrave Macmillan.

Weiste, E., \& Peräkylä, A. (2013). A comparative conversation analytic study of formulations in psychoanalysis and cognitive psychotherapy. Research on Language and Social Interaction, 46(4), 299-321. https://doi.org/10.1080/08351813.2013.839093.

Whitworth, L., Kimsey-House, K., Kimsey-House, H., \& Sandahl, P. (1998). Co-active coaching. New skills for coaching people toward success in work and life. Davis-Black.

Publisher's Note Springer Nature remains neutral with regard to jurisdictional claims in published maps and institutional affiliations. 